\title{
Polydopamine-coated UiO-66 nanoparticles loaded with perfluorotributylamine/ tirapazamine for hypoxia-activated osteosarcoma therapy
}

\author{
Hongfang Chen ${ }^{1 \dagger}$, You Fu ${ }^{2 \dagger}$, Kai Feng ${ }^{3 \dagger}$, Yifan Zhou ${ }^{1}$, Xin Wang ${ }^{1}$, Haohan Huang ${ }^{1}$, Yan Chen ${ }^{1}$, Wenhao Wang ${ }^{4}$,
} Yuanjing $\mathrm{Xu}^{5}$, Haijun Tian ${ }^{1 *}$, Yuanqing Mao ${ }^{1 *}$, Jinwu Wang ${ }^{1 *}$ and Zhiyuan Zhang ${ }^{2}$

\begin{abstract}
Background: Hypoxia is a characteristic of solid tumors that can lead to tumor angiogenesis and early metastasis, and addressing hypoxia presents tremendous challenges. In this work, a nanomedicine based on oxygen-absorbing perfluorotributylamine (PFA) and the bioreductive prodrug tirapazamine (TPZ) was prepared by using a polydopamine (PDA)-coated UiO-66 metal organic framework (MOF) as the drug carrier.

Results: The results showed that TPZ/PFA@UiO-66@PDA nanoparticles significantly enhanced hypoxia, induced cell apoptosis in vitro through the oxygen-dependent HIF-1a pathway and decreased oxygen levels in vivo after intratumoral injection. In addition, our study demonstrated that TPZ/PFA@UiO-66@PDA nanoparticles can accumulate in the tumor region after tail vein injection and effectively inhibit tumor growth when combined with photothermal therapy (PTT).TPZ/PFA@UiO-66@PDA nanoparticles increased HIF-1a expression while did not promote the expression of CD31 in vivo during the experiment.

Conclusions: By using TPZ and PFA and the enhanced permeability and retention effect of nanoparticles, TPZ/PFA@ UiO-66@PDA can target tumor tissues, enhance hypoxia in the tumor microenvironment, and activate TPZ. Combined with PTT, the growth of osteosarcoma xenografts can be effectively inhibited.
\end{abstract}

Keywords: Metal-organic framework (MOF), Tumor hypoxia, Photothermal therapy (PTT), Osteosarcoma

\section{Introduction}

The fast growth and insufficient nutrient supply of solid tumors result in tumor hypoxia $[1,2]$, which can not only promote tumor angiogenesis and metastasis [3] but can also lead to therapy resistance $[4,5]$. To treat hypoxic

\footnotetext{
*Correspondence: haijuntianmd@gmail.com; yuanqingmao@163.com; wangjw-team@shsmu.edu.cn

${ }^{\dagger}$ Hongfang Chen, You Fu and Kai Feng contributed equally to this manuscript

${ }^{1}$ Shanghai Key Laboratory of Orthopaedic Implants, Department of Orthopaedic Surgery, Shanghai Ninth People's Hospital, Shanghai Jiao Tong University School of Medicine, Shanghai 200011, China

Full list of author information is available at the end of the article
}

tumors, two methods are highlighted: tumor oxygenation and tumor deoxygenation [6-8]. For tumor oxygenation, various oxygen-delivery strategies have been explored to alleviate tumor hypoxia $[9,10]$. However, the therapeutic effect of these solutions can be unsatisfactory due to premature $\mathrm{O}_{2}$ leakage, low $\mathrm{O}_{2}$ production, and insufficient neovascularization and blood flow in tumor tissues. Hence, strategies focused on tumor deoxygenation combined with hypoxia-activated prodrugs have also been developed for hypoxic tumor treatment.

Tirapazamine (TPZ) is a bioreductive prodrug that can be transformed into tumor-toxic benzotriazinyl (BTZ) 
by hypoxia [11]. However, a single use of TPZ can elicit only a marginal effect on tumor growth because a limited amount of BTZ is generated from TPZ due to restricted penetration into the tumor and insufficient tumor hypoxia [12]. Accordingly, many studies have focused on creating a penetrable and hypoxic intratumor microenvironment to enhance the toxicity of hypoxia-sensitive prodrugs [13-15]. Perfluorotributylamine (PFA) is a clinically approved perfluorocarbon that can enhance tumor permeability and deoxygenation due to its platelet inhibition and oxygen-absorbing effects. Wang et al. [16] demonstrated that PFA can establish a long-lasting favorable tumor microenvironment to enable hypoxia-sensitive prodrug delivery and activation.

Metal organic frameworks (MOFs) are a new class of topologically organic-inorganic hybrid materials constructed by inorganic nodes and organic ligands via spontaneous self-assembly [17] and bear multiple complexing functions [18]. The large family of organic linkers available [19] present high and regular porosities, allowing MOFs to be used for gas adsorption, separation and storage, molecular sensing, drug delivery, pollution management, catalysis, and energy storage [20-23]. MOFs can simultaneously exhibit hydrophobic and hydrophilic properties and have a tunable connectivity and pore size; thus, MOFs can be specifically adapted to the physicochemical properties of each drug and its medical application [24, 25]. For example, glucose/oxygen depletion agents, such as glucose oxidase and gold nanoparticles (NPs), can be integrated into MOF NPs to promote intracellular Fenton-like reactions and effectively suppress tumor growth $[26,27]$. Since the pore size, pore shape and pore chemistry of MOF materials can be precisely controlled, numerous MOFs have been developed for gas adsorption. Chen et al. [28] synthesized a novel mesoporous MOF constructed from a trigonal tetrazolate ligand and $\mathrm{Cu}^{2+}$, which demonstrated exceptionally high uptake capacities for fluorocarbons due to its high porosity. Shen et al. [29] demonstrated that sulfone-based 2D fluorinated MOFs showed a high adsorption capacity with high selectivity and stability, enabling the separation of acetylene from ethylene. The characteristics of MOF materials make them appealing drug carriers for cancer treatment. In this study, we used PFA-loaded MOF NPs as an oxygen absorbent to promote tumor hypoxia. Since hypoxia is often associated with therapeutic resistance, the combination of other therapeutic methods was considered. Photothermal therapy (PTT) has been developed in the past decade and is regarded as a promising strategy due to its noninvasiveness, limited side effects, and high ablation efficacy [30]. PTT is utilized to further enhance tumor permeability and the killing of drug-resistant tumor cells.
In this study, we developed a microporous MOFbased nanocarrier coated with polydopamine (PDA) and encapsulating PFA and TPZ for hypoxia-activated osteosarcoma therapy. As illustrated in Scheme 1, UiO-66 was chosen as a nanocarrier to encapsulate PFA and the prodrug TPZ because of its large surface area and high porosity. Then, the fabricated MOF NPs were further coated with PDA to obtain TPZ/PFA@UiO-66@PDA. After intravenous injection, TPZ/PFA@UiO-66@PDA effectively accumulated in tumor tissue via the typical enhanced permeability and retention (EPR) effect. The PFA in TPZ/PFA@UiO-66@PDA efficiently absorbed oxygen to aggravate the hypoxic tumor microenvironment. Enhanced tumor hypoxia could then activate and transform the prodrug TPZ into highly cytotoxic radicals to induce tumor cell apoptosis. Moreover, the accumulation and internalization of PDA further enhanced tumor permeability and the killing of drug-resistant tumor cells under $808 \mathrm{~nm}$ laser irradiation. In this way, TPZ/PFA@ UiO-66@PDA NPs achieved synergistic efficacy between hypoxia-activated bioreductive prodrug therapy and PTT to effectively suppress tumor growth.

\section{Experiments}

\section{Ethics statement}

All experiments involving animals were reviewed and approved by the Ethical Committee of Shanghai Ninth People's Hospital in Shanghai, China.

\section{Synthesis of drug-loaded UiO-66}

UiO-66 was synthesized based on a previously reported method [31-33]. $\mathrm{ZrCl}_{4}$ (1 mmol or $0.2332 \mathrm{~g}$ ) and TPA $(1 \mathrm{mmol}$ or $0.161 \mathrm{~g})$ were dissolved in $50 \mathrm{~mL}$ of DMF solution and then transferred to a $100 \mathrm{~mL}$ autoclave. The autoclave was sealed and heated in an oven at $120{ }^{\circ} \mathrm{C}$ for $48 \mathrm{~h}$. The resulting white powder was washed several times with methanol after cooling and then dried at $100{ }^{\circ} \mathrm{C}$ for $12 \mathrm{~h}$ under vacuum. Finally, the powder was stored for further use. To load the anticancer drugs into the MOF, $100 \mathrm{mg}$ of UiO-66 and $50 \mathrm{mg}$ of TPZ were poured into a mixture of $5 \mathrm{~mL}$ of DMSO and $5 \mathrm{ml}$ of ethanol, stirred for $12 \mathrm{~h}$, and then centrifuged at $5000 \mathrm{rpm}$ for $20 \mathrm{~min}$. The mixture was washed with ethanol twice and freeze dried. Then, $100 \mathrm{mg}$ of the mixture was poured into $0.2 \mathrm{~mL}$ of PFA, placed on a shaker for $3 \mathrm{~h}$, and centrifuged. Next, $100 \mathrm{mg}$ of the mixture and $35 \mathrm{mg}$ of PDA were poured into 20 $\mathrm{mL}$ of hydrochloric acid with a $\mathrm{pH}$ of 8.5 , stirred for $45 \mathrm{~min}$, and then centrifuged at $5000 \mathrm{rpm}$ for $20 \mathrm{~min}$. After freeze-drying, TPZ/PFA@UiO-66@PDA NPs were dispersed in phosphate-buffered saline (PBS) for further use. For IR780-doped PFA/TPZ@UiO-66@ PDA NPs, IR780 (5 mg) and PFA/TPZ@UiO-66@PDA 


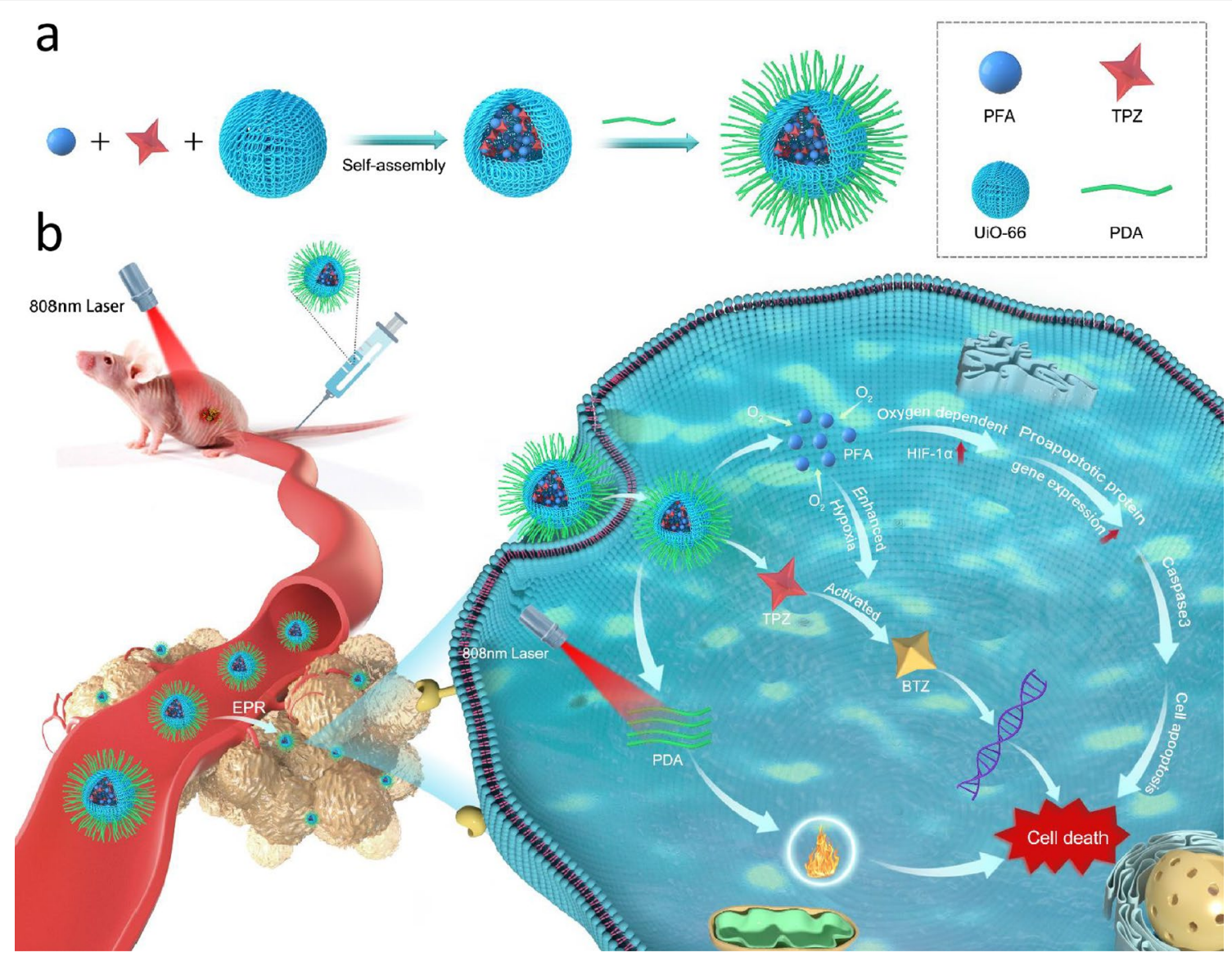

Scheme 1 Schematic illustration of $\mathbf{a}$ the preparation of TPZ/PFA@UiO-66@PDA NPs and $\mathbf{b}$ tail vein-injected TPZ/PFA@UiO-66@PDA NPs combined with laser radiation for hypoxia-activated osteosarcoma therapy

(50 mg) were mixed in ethanol for $24 \mathrm{~h}$. After centrifugation, the mixture was dispersed in $20 \mathrm{~mL}$ Tris- $\mathrm{HCl}$ $(\mathrm{pH}=8.5)$ and stirred for $45 \mathrm{~min}$. Then, the mixture was centrifuged and freeze dried for further use.

\section{General characterization}

The crystallinity and surface morphology of the prepared UiO-66 MOF were detected using X-ray diffraction (XRD, D8 VENTURE), Brunauer-Emmett-Teller (BET) surface area analysis (Autosorb-IQ3), scanning electron microscopy (SEM, MIRA3), and transmission electron microscopy (TEM, Talos F200X G2). UiO66 and UiO-66@PDA were characterized by Fourier Transform Infrared Spectroscopy (FTIR). The drug loading efficiency of UiO-66, TPZ@UiO-66, and TPZ/ PFA@UiO-66 was analyzed via thermogravimetric analysis (TGA, Pyris 1). The particle size distribution of UiO-66, UiO-66@PDA and TPZ/PFA@UiO-66@PDA were detected by dynamic light scattering (DLS).

\section{Cell culture}

143B cells were obtained from ATCC (Manassas, VA) and used in this study. The cells were cultured in Dulbecco's minimum essential medium (DMEM) supplemented with $10 \%$ fetal bovine serum (FBS), penicillin $(100 \mathrm{U} / \mathrm{mL})$, and streptomycin $(100 \mathrm{mg} / \mathrm{mL})$ in a humidified atmosphere containing $5 \% \mathrm{CO}_{2}$ at $37^{\circ} \mathrm{C}$. The culture medium was changed every 2 days. Cells were passaged upon reaching $80-90 \%$ confluence.

\section{Cell viability}

Cell viability was assessed using a live/dead assay (Thermo Fisher Scientific). 143B cells were divided into the following groups: control, TPZ $\left(40 \mu \mathrm{g} \mathrm{mL}{ }^{-1}\right)$, TPZ@ UiO-66 (40 $\left.\mu \mathrm{g} \mathrm{mL} \mathrm{m}^{-1}\right), \mathrm{TPZ} / \mathrm{PFA} @ U i O-66 @ P D A(40 \mu \mathrm{g}$ $\mathrm{mL}^{-1}$ ), and TPZ/PFA@UiO-66@PDA+808 nm laser radiation. Each group was cultured for $3 \mathrm{~h}$ in 24-well plates at a density of $2.5 \times 10^{4}$ cells/well. For group 5, cells were irradiated with an $808 \mathrm{~nm}$ laser for $5 \mathrm{~min}$. Then, all samples were stained in a solution containing 
calcein-AM ( $2 \mu \mathrm{M}$ in PBS) and ethidium homodimer (EthD)-1 $(4 \mu \mathrm{M})$ for $30 \mathrm{~min}$ and examined using a fluorescence microscope (Leica, Wetzlar, Germany). Live cells were stained green, and dead cells were stained red.

\section{In vitro hypoxia and apoptosis analysis using flow cytometry (FCM)}

143B cells were seeded into 6-well plates and cultured. When a confluence of $70-80 \%$ was reached, the cells were washed with PBS and grouped as previously stated. For apoptosis analysis, $5 \mu \mathrm{L}$ of annexin V-FITC and $5 \mu \mathrm{L}$ of propidium iodide (PI) were added to all wells and then incubated with the cells for $15 \mathrm{~min}$ at room temperature in the dark according to the manufacturer's instructions (Thermo Scientific, Waltham, MA, USA). For hypoxia analysis, cell suspensions of each group were treated with $100 \mu \mathrm{M}$ Hypoxyprobe-1 (pimonidazole hydrochloride) for $1 \mathrm{~h}$. The cells were collected, fixed and stained with anti-pimonidazole antibody (1:200, clone 4.3.11.3; FITCMAb1). The fluorescence intensity of all samples was immediately analyzed via flow cytometry.

\section{Quantitative real-time PCR}

To examine the effects of the NPs on oxygen-dependent signaling in 143B cells at the gene level, 143B cells were seeded and grouped as follows: Control, TPZ $(40 \mu \mathrm{g}$ $\left.\mathrm{mL}^{-1}\right)$, TPZ@UiO-66 (40 $\left.\mu \mathrm{g} \mathrm{mL}^{-1}\right)$, and TPZ/PFA@ UiO-66@PDA $\left(40 \mu \mathrm{g} \mathrm{mL}^{-1}\right)$. All groups were cultured in 6-well plates at a density of $1 \times 10^{5}$ cells/well for $3 \mathrm{~h}$. Total RNA was isolated using an E.Z.N.A. ${ }^{\circledR}$ HP Total RNA Kit (Omega Bio-Tek, Norcross, GA, USA). One microgram of RNA was reverse transcribed using a Prime-Script RT reagent kit (Takara, Shiga, Japan). Real-time PCR was performed in a 7300 Real-Time PCR system using SYBR ${ }^{\circledR}$ Premix Ex $\mathrm{Taq}^{\mathrm{TM}}$ (Takara, Shiga, Japan). The primer sequences used in this study are listed in Table 1. Glyceraldehyde 3-phosphate dehydrogenase 1 (GAPDH) was evaluated for each RT reaction as a standard. The $\Delta \Delta \mathrm{CT}$ method was used to analyze relative gene expression.

\section{Western blotting analysis}

To examine the effects of NPs on HIF- $1 \alpha$ in 143B cells at the protein level, 143B cells were seeded and grouped as follows: Control, TPZ ( $\left.40 \mu \mathrm{g} \mathrm{mL}{ }^{-1}\right)$, TPZ@UiO-66 (40 $\mu \mathrm{g}$ $\left.\mathrm{mL}^{-1}\right)$, and TPZ/PFA@UiO-66@PDA $\left(40 \mu \mathrm{g} \mathrm{mL}^{-1}\right)$. All groups were cultured for $3 \mathrm{~h}$ in 6-well plates at a density of $1 \times 10^{5}$ cells/well. The cells were washed three times with PBS and then lysed with RIPA buffer $(150 \mathrm{mM}$ $\mathrm{NaCl}, 1 \%$ sodium deoxycholate, $0.1 \%$ SDS, $50 \mathrm{mM}$ Tris$\mathrm{HCl} \mathrm{pH} \mathrm{7.4,} 1$ mM EDTA, 1 mM PMSF, and 1\% Triton $\mathrm{X}-100)$ supplemented with protease inhibitors and phosphatase inhibitors for $30 \mathrm{~min}$ at $4{ }^{\circ} \mathrm{C}$. A total of $20-30 \mathrm{mg}$ of protein was separated via $10 \%$ sodium dodecyl
Table 1 Sense and anti-sense primers for real-time PCR

\begin{tabular}{|c|c|c|}
\hline Gene & Forward $\left(5^{\prime}\right.$ to $\left.3^{\prime}\right)$ & Reverse $\left(5^{\prime}\right.$ to $\left.3^{\prime}\right)$ \\
\hline HIF-1a & $\begin{array}{l}\text { AGTTCCGCAAGCCCTGAA } \\
\text { AGC }\end{array}$ & $\begin{array}{l}\text { GCAGTGGTAGTGGTGGCA } \\
\text { TTAGC }\end{array}$ \\
\hline HIF-1 $\beta$ & $\begin{array}{l}\text { GCTACTGCTAAGACTCGT } \\
\text { ACTT }\end{array}$ & $\begin{array}{l}\text { ACGATTGGTGAGACTAGG } \\
\text { GTAG }\end{array}$ \\
\hline p300 & $\begin{array}{l}\text { GTTCCTTCCTCAGACTCA } \\
\text { GTTC }\end{array}$ & $\begin{array}{l}\text { CATTATAGGAGAGTTCAC } \\
\text { CGGG }\end{array}$ \\
\hline CBP & $\begin{array}{l}\text { CAAGGAGGTCTTCTTCGT } \\
\text { GATC }\end{array}$ & $\begin{array}{l}\text { CTCGTTGCAGGTGTAGAC } \\
\text { AAAG }\end{array}$ \\
\hline BAX & $\begin{array}{l}\text { CGAACTGGACAGTAACAT } \\
\text { GGAG }\end{array}$ & $\begin{array}{l}\text { CAGTTTGCTGGCAAAGTA } \\
\text { GAAA }\end{array}$ \\
\hline BAK & AGAGATGGTCACCTTACCTCT & $\begin{array}{l}\text { GGTCTGGAACTCTGAGTC } \\
\text { ATAG }\end{array}$ \\
\hline BID & CTGTGAACCAGGAGTGAGTC & GTAGGTTTGTGATGCACTCATC \\
\hline Caspase-3 & $\begin{array}{l}\text { CCAAAGATCATACATGGA } \\
\text { AGCG }\end{array}$ & CTGAATGTTTCCCTGAGGTTTG \\
\hline GAPDH & CAGCGACACCCACTCCTC & TGAGGTCCACCACCCTGT \\
\hline
\end{tabular}

sulfate-polyacrylamide gel electrophoresis and electrotransferred onto nitrocellulose membranes. A rabbit polyclonal anti-HIF-1 $\alpha$ antibody (Cell Signaling Technology) was used as the primary antibody. GAPDH antibody (Cell Signaling Technology) was used to normalize protein loading. The protein bands were visualized using an Odyssey Infrared Imaging System (LI-COR Biosciences, Lincoln, NE, USA).

\section{Implantation of tumor cells and test materials}

Six-week-old male BALB/c nude mice were purchased from Shanghai Super-B\&K Laboratory Animal Corp. Ltd. A total of $1.0 \times 10^{6} 143 \mathrm{~B}$ cells in a volume of $100 \mu \mathrm{L}$ that contained $50 \mu \mathrm{L}$ of culture medium and $50 \mu \mathrm{L}$ of growth factor-reduced Matrigel (BD Biosciences, San Jose, CA, USA) were subcutaneously injected into the lower left flanks of the mice. When the tumor size reached approximately $80 \mathrm{~mm}^{3}$, the mice were divided into five groups as follows: Group 1, Control group treated with saline only; Group 2, TPZ (10 mg kg-1, $100 \mu \mathrm{L})$; Group 3, TPZ@ UiO-66 (10 mg kg-1, $100 \mu \mathrm{L})$; Group 4, TPZ/PFA@ UiO-66@PDA (10 mg kg $\left.{ }^{-1}, 100 \mu \mathrm{L}\right)$; and Group 5, TPZ/ PFA@UiO-66@PDA+808 nm laser radiation. The treatment or saline was intravenously injected into the mice on days 0,4 and 8 . The tumors in group 5 were irradiated three times $\left(808 \mathrm{~nm}, 1.5 \mathrm{~W} \mathrm{~cm}^{-2}\right)$ for $5 \mathrm{~min}$ at $24 \mathrm{~h}$ after intravenous injection. The mice were anesthetized and intraperitoneally injected with D-luciferin $(150 \mathrm{mg} /$ kg, Caliper Life Sciences, Hopkinton, MA). Twenty minutes after the injection, all mice were photographed using an IVIS cooled CCD camera (Xenogen, Alameda, CA). The mice were imaged on days 0,8 and 16 . The weights 


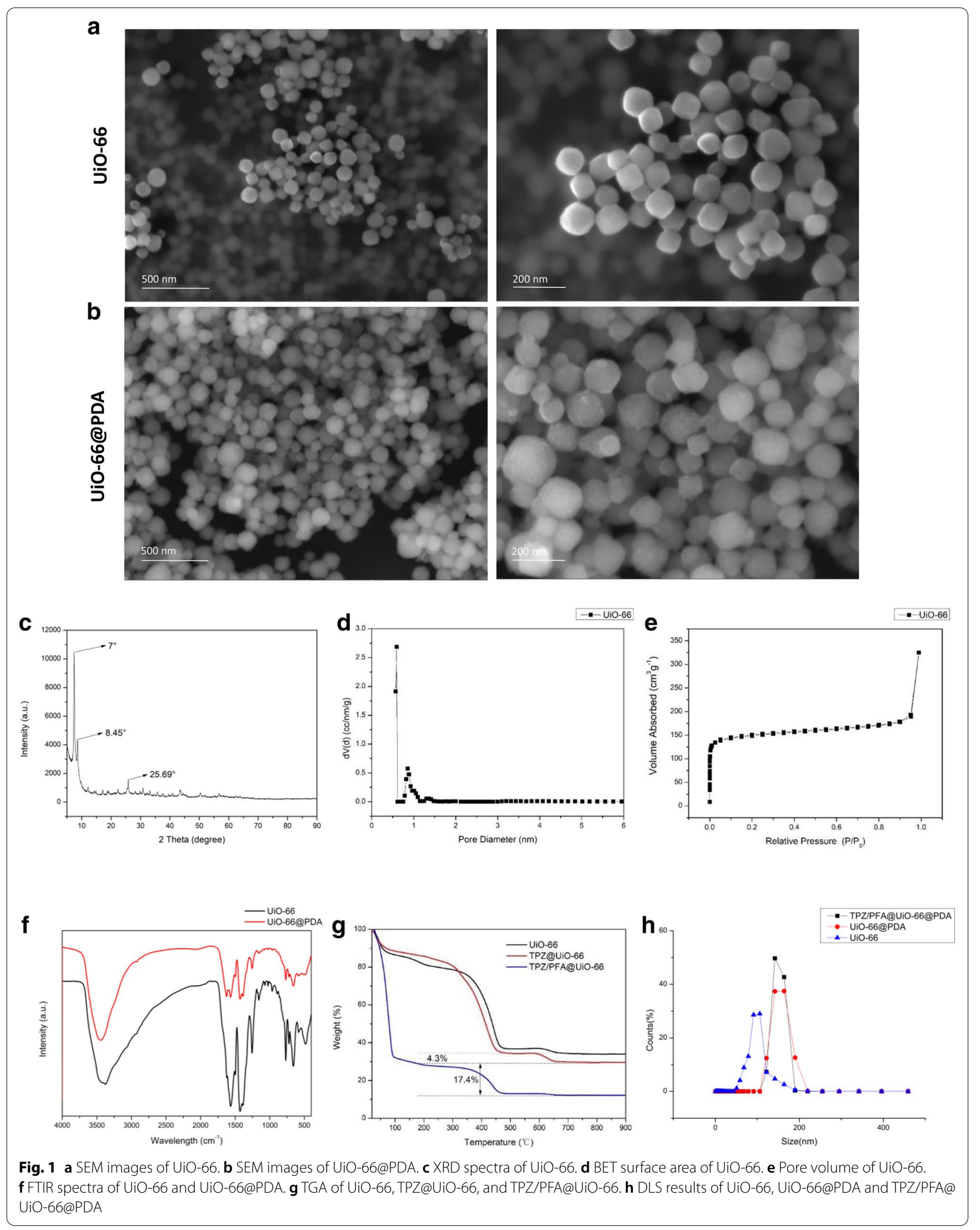



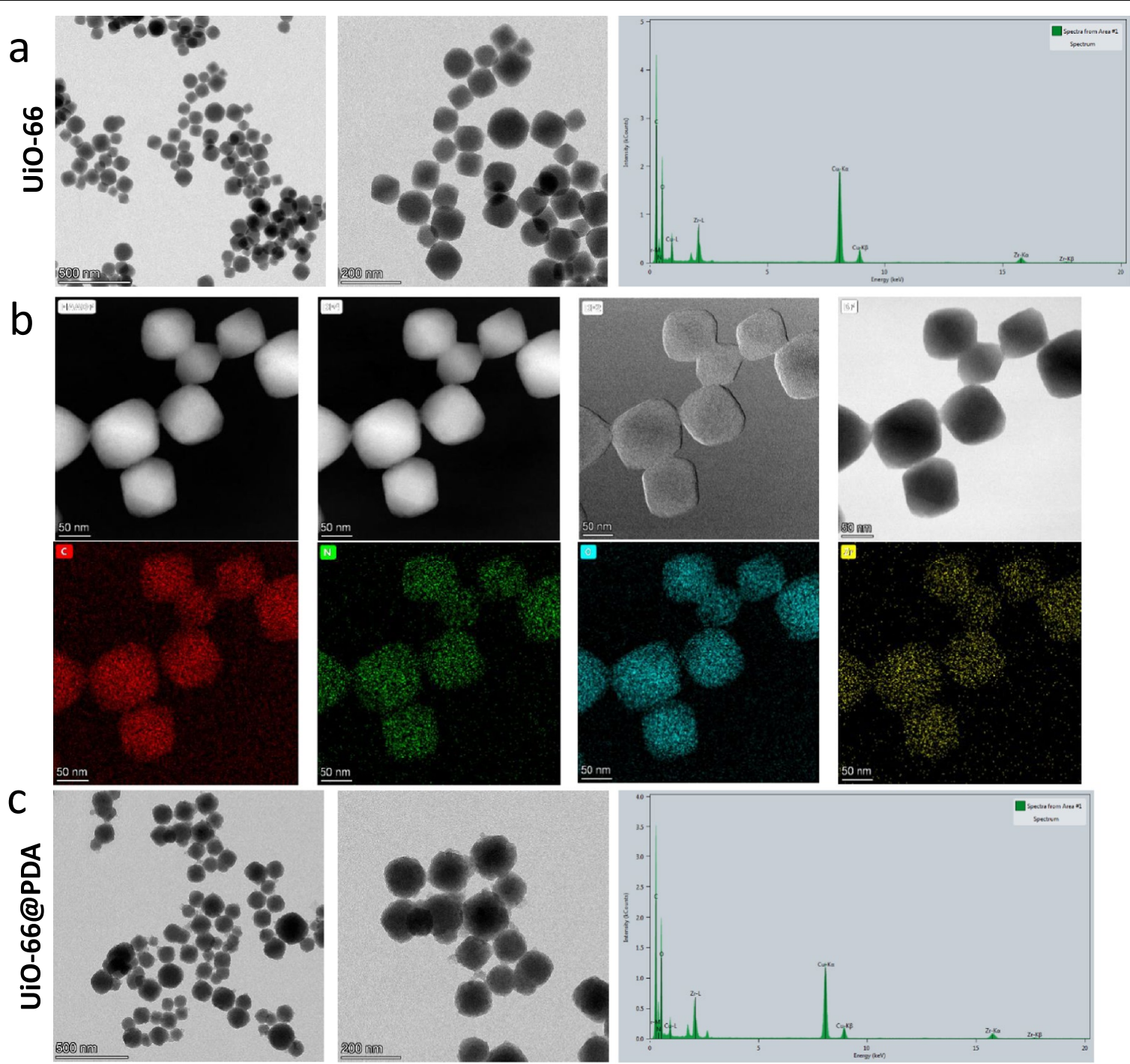

d
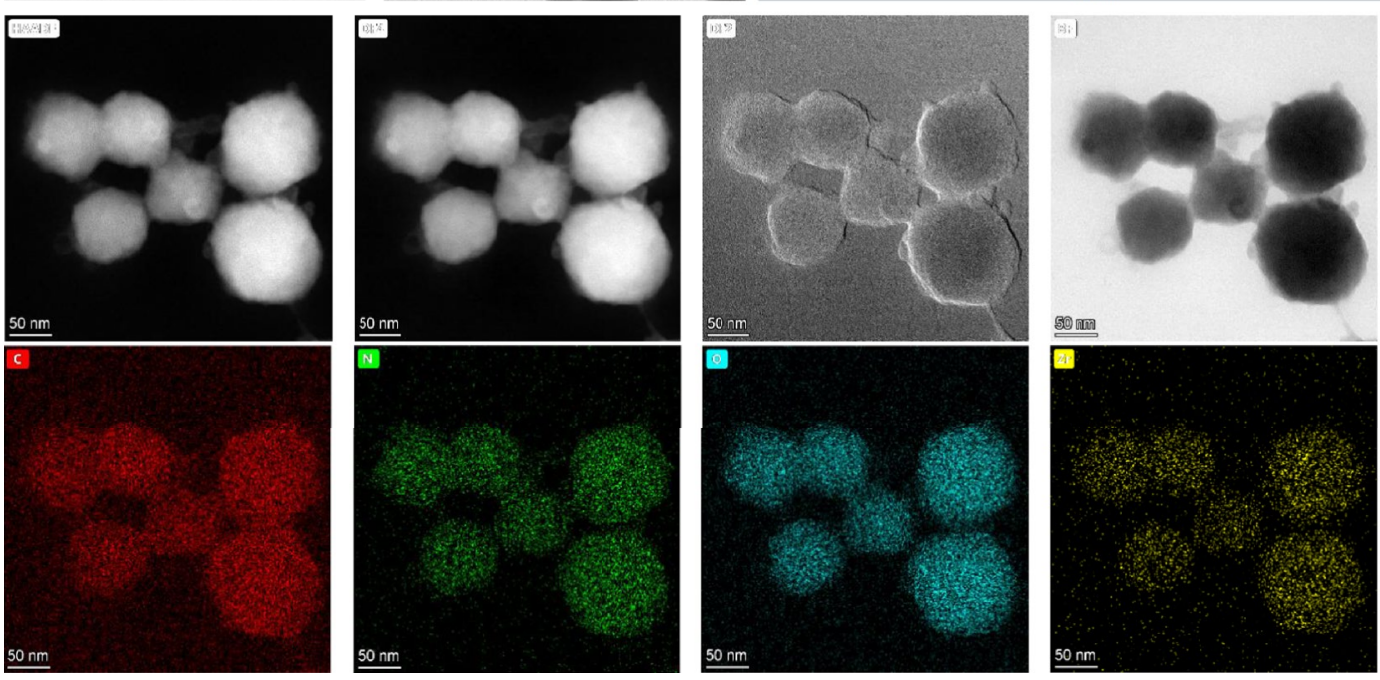

Fig. 2 a TEM and EDS images of UiO-66. b Elemental mapping images of UiO-66. cTEM and EDS images of UiO-66@PDA. d Elemental mapping images of UiO-66@PDA 
of all mice were measured every other day. The dissected tumor weight was measured using a precision electronic balance.

\section{In vivo photoacoustic (PA) imaging}

For in vivo PA imaging, 143B tumor-bearing mice were intratumorally injected with NPs $\left(10 \mathrm{mg} \mathrm{kg}^{-1}, 100 \mu \mathrm{L}\right)$. The intensities of PA signals and PA images of tumorbearing mice were measured and recorded at different time points $(0,0.5,1,2$, and $3 \mathrm{~h})$. The intensities of the PA signals and PA images from mice injected with saline were used as the control. The imaging parameters were set as follows: PA gain, $47 \mathrm{~dB}$, and $2 \mathrm{D}$ gain, $20 \mathrm{~dB}$.

\section{Histology and immunohistochemistry}

143B cells were cultured in $10 \mathrm{~mm}$ confocal dishes at a density of $1 \times 10^{5}$ cells/dish. After cell attachment, the dishes were treated and grouped as follows: Control, TPZ, TPZ@UiO-66, and TPZ/PFA@UiO-66@PDA. Each group was incubated for $3 \mathrm{~h}$. Then, all dishes were rinsed with PBS, fixed for $15 \mathrm{~min}$ at room temperature in $4 \%$ paraformaldehyde (Sigma-Aldrich, St. Louis, MO, USA), and permeabilized with $0.2 \%$ Triton X-100. After blocking with $0.5 \% \mathrm{BSA}$ and $1 \% \mathrm{FBS}$, the cells were stained with anti-HIF-1 $\alpha$ antibody, followed by Alexa 488-conjugated secondary antibody. Finally, the samples were washed with PBS for direct observation under confocal laser scanning microscopy (Nikon Eclipse CI, Tokyo, Japan). The subcutaneous tumor specimens were fixed at $4{ }^{\circ} \mathrm{C}$ in $4 \%$ paraformaldehyde (Sigma-Aldrich, St. Louis, $\mathrm{MO}, \mathrm{USA}$ ) and washed in running water overnight before being embedded in paraffin. Hematoxylin-eosin (H\&E) staining was conducted on $5-\mu \mathrm{m}$ sections for histological analyses. Immunohistochemistry was performed to detect PCNA, CD31, HIF-1 $\alpha$ and pimonidazole in tumor sections. Supplementary methods were provided in Additional file 1.

\section{Statistical analysis}

In this article, all data are presented as the mean \pm standard deviation (SD) and were analyzed with SPSS version 22.0 (IBM, Armonk, NY). Comparisons of two or more groups were performed using one-way analysis of variance (ANOVA) with subsequent Tukey-Kramer comparisons. Student's t-test was used for comparisons involving two groups. $\mathrm{P}<0.05$ was considered statistically significant.

\section{Results and discussion Characterization of prepared UiO-66 and UiO-66/PDA}

XRD and PXRD were employed to characterize the structure of the synthesized UiO-66. As shown in Fig. 1c, the XRD pattern of UiO-66 has three peaks at $7^{\circ}, 8.45^{\circ}$, and $25.69^{\circ}$ [33, 34]. Additional file 2: Fig. S1 showed the PXRD pattern of UiO-66. The Bragg diffraction peaks of the samples indicated that the NPs maintained a wellordered pore structure, which was beneficial for drug delivery. BET analysis demonstrated that the UiO-66 samples displayed typical Type-I gas sorption isotherms. UiO-66 exhibited a BET surface area of $655.89 \mathrm{~m}^{2} \mathrm{~g}^{-1}$, which indicated that the NPs were suitable for drug loading (Fig. 1d). Figure 1e showed that the pore volume was $0.274 \mathrm{cc} / \mathrm{g}$. Figure $1 \mathrm{f}$ demonstrates the FTIR spectra of UiO-66 and UiO-66@PDA. The shapes of UiO-66 and UiO-66@PDA were characterized by SEM and TEM (Figs. 1a, b and 2a and c). Figures 1a and 2a showed the morphology of the prepared UiO-66. The TEM image demonstrated that the sizes of the most UiO-66 crystals were between 80 and $160 \mathrm{~nm}$. Elemental mapping images revealed that $\mathrm{Zr}$ was dispersed homogeneously in UiO-66 (Fig. 2b). The SEM and TEM images of TPZ/ PFA@UiO-66@PDA were provided in Additional file 3: Fig. S2. After modification with PDA, the UiO-66@PDA composite displayed rough surfaces (Fig. 2c). Figure 2d showed the elemental mapping of UiO-66@PDA. These results indicated successful fabrication of UiO-66@PDA NPs and that the coating process did not change the elemental distribution of UiO-66. TPZ/PFA@UiO-66@PDA NPs remained stable in PBS or culture media for at least $24 \mathrm{~h}$ (Additional file 4: Fig. S3). Figure 1g showed that the mass fraction of TPZ in TPZ@UiO-66 was 4.3\%, and the mass fraction of PFA in TPZ/PFA@UiO-66 was 17.4\%, and the drug-loading efficiency for TPZ and PFA of UiO66 was 8.6 and $7.062 \%$ respectively. Additional file 5: Fig. S4 demonstrated the standard calibration curve of TPZ and the in vitro release curve of TPZ from TPZ@UiO-66 and TPZ/PFA@UiO-66@PDA.And TPZ/PFA@UiO-66@ PDA NPs showed a slow release pattern of TPZ. DLS results of UiO-66, UiO-66@PDA and TPZ/PFA@UiO66@PDA indicated that the particle size increased after

(See figure on next page.)

Fig. 3 a CLSM images of 143B cells incubated with PFA/TPZ@UiO-66@PDA NPs for 3 h. Red fluorescence is attributed to IR780-doped PFA/ TPZ@UiO-66@PDA NPs. Nuclei were stained blue with DAPI. Cytoskeletons were stained green with FITC-Phalloidin. b Cellular uptake behaviors of PFA/TPZ@UiO-66@PDA NPs determined by flow cytometry. c Hypoxia assessment via flow cytometry analysis of $143 \mathrm{~B}$ cells under different treatments. $\mathbf{d}$ Apoptosis analysis of 143B cells after different treatments for $3 \mathrm{~h}$. $\mathbf{e}$ The quantitative results for 143B cells obtained using pimonidazole hydrochloride. $\mathrm{fLive/dead} \mathrm{staining} \mathrm{of} \mathrm{143B} \mathrm{cells} \mathrm{after} \mathrm{incubation} \mathrm{with} \mathrm{different} \mathrm{treatments} \mathrm{for} 3 \mathrm{~h}$. Viable cells were stained with calcein-AM (green), and dead/late apoptotic cells were stained with ethidium homodimer (EthD)- 1 (red). $\mathbf{g}$ The quantitative flow cytometry results for cell apoptosis analysis. Asterisks indicate significant differences (scale bar, $100 \mu \mathrm{m})\left({ }^{*} \mathrm{P}<0.05,{ }^{* *} \mathrm{P}<0.01,{ }^{* * *} \mathrm{P}<0.001,{ }^{* * * *} \mathrm{P}<0.0001\right.$ ) 
a

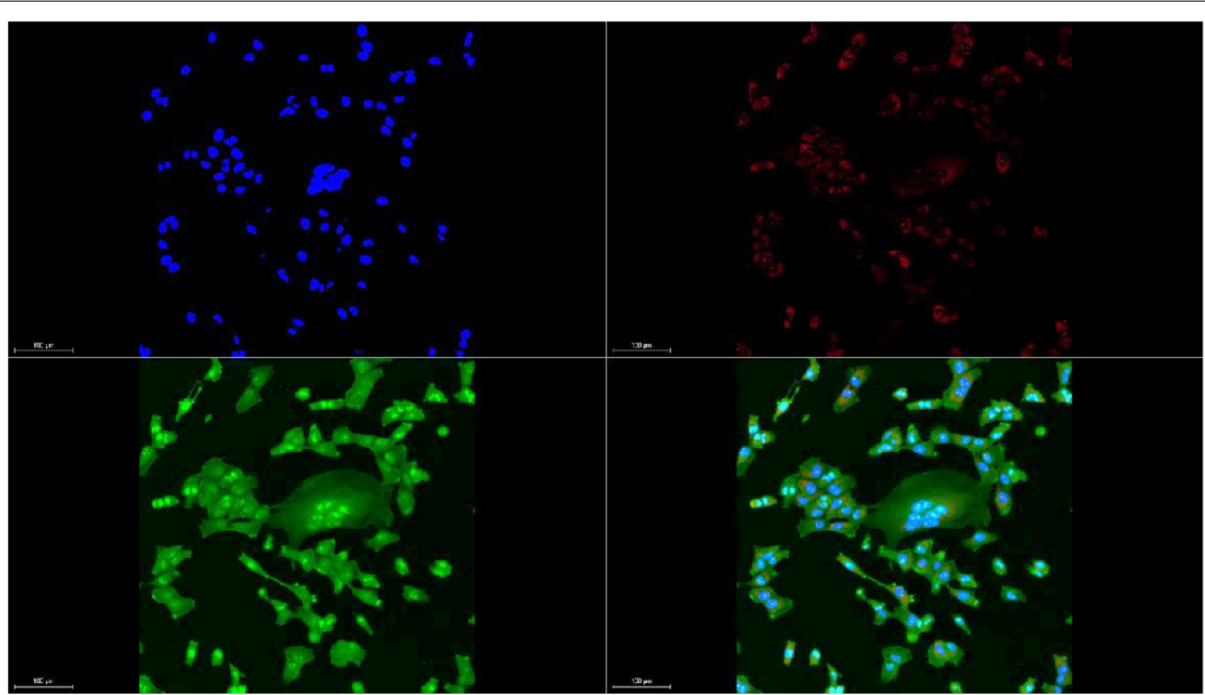

b
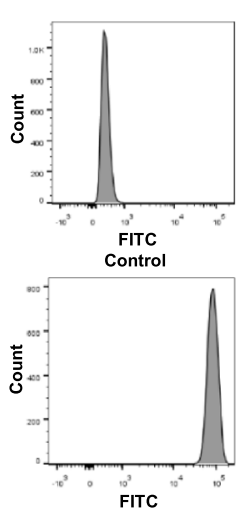

FITC
TPZPFA@UiO-66@PDA

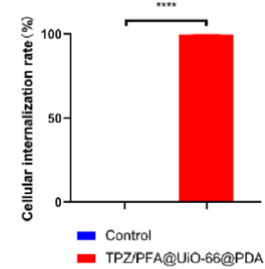

C
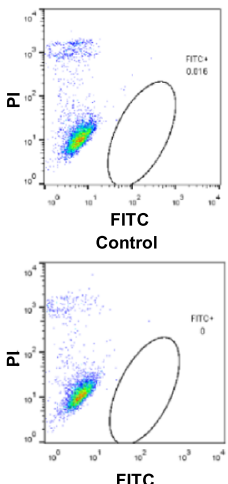

TPZ@UiO-66

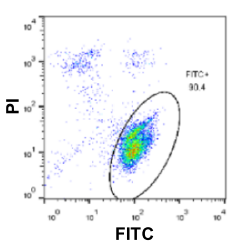

TPZ/PFA@UiO-66@PDA

e

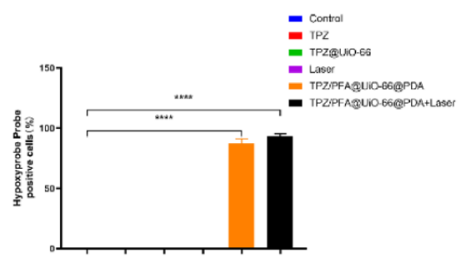

g

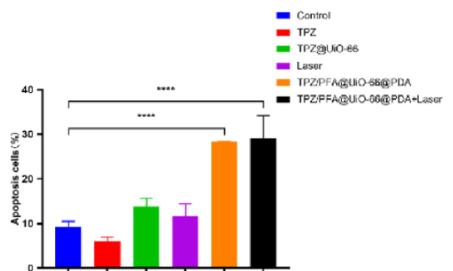

d
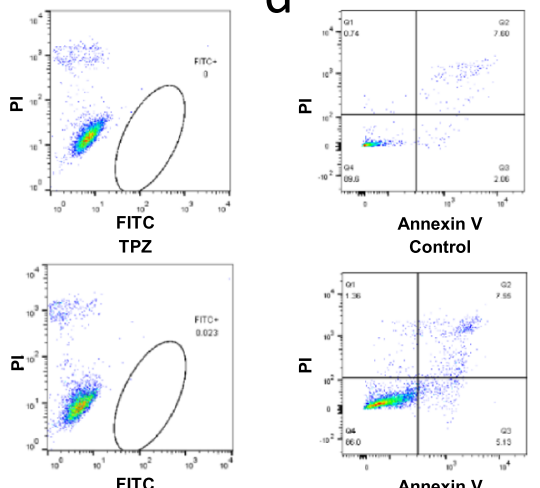

FITC
Laser

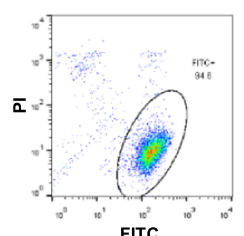

TPZ/PFA@UiO-66@PDA+Laser

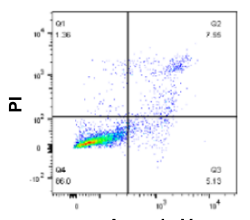

Annexin V

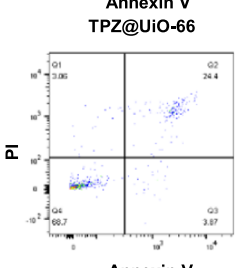

Annexin $\mathrm{V}$ TPZ/PFA@UiO-66@PDA
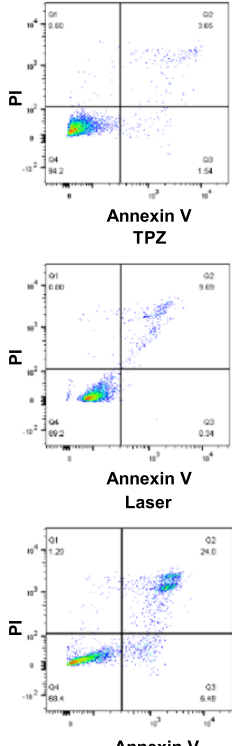

TPZ/PFA@UiO-66@PDA+Laser

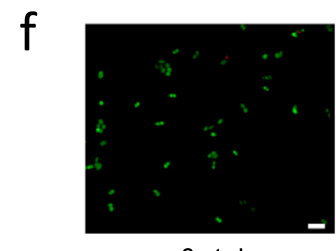

Control

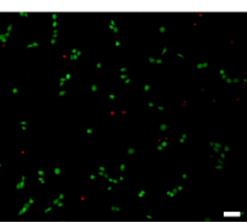

TPZ

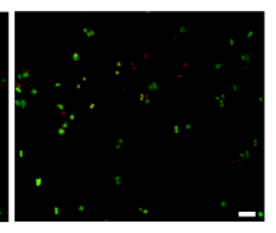

TPZ@UiO-66

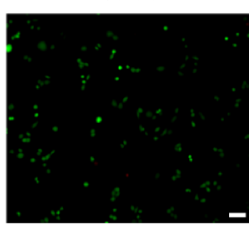

Laser

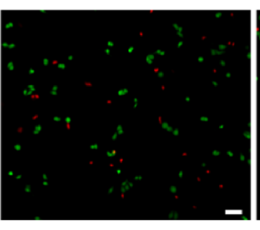

TPZ/PFA@UiO-66@PDA

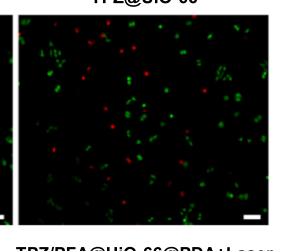

Fig. 3 (See legend on previous page.) 
PDA coating and drug loading (Fig. 1h). TPZ/PFA@UiO66@PDA NPs remained stable in PBS or culture media for at least $24 \mathrm{~h}$ (Fig. S3).

\section{Evaluation of TPZ/PFA@UiO-66@PDA in cell culture}

Additional file 6: Fig. S5 indicated that UiO-66 showed no obvious toxicity in both $143 \mathrm{~B}$ and rat bone marrow stem cells with different concentration of UiO-66 $(0 \mu \mathrm{g}$ $\mathrm{mL}^{-1}, 10 \mu \mathrm{g} \mathrm{mL}{ }^{-1}, 20 \mu \mathrm{g} \mathrm{mL}{ }^{-1}, 40 \mu \mathrm{g} \mathrm{mL}^{-1}, 80 \mu \mathrm{g}$ $\mathrm{mL}^{-1}$ ).To evaluate the cellular uptake behavior, TPZ/ PFA@UiO-66@PDA NPs were incubated with 143B cells for $3 \mathrm{~h}$. As illustrated in Fig. 3a, the red fluorescence in the cells suggested strong cellular internalization. Flow cytometry analysis also confirmed the high uptake of TPZ/PFA@UiO-66@PDA NPs (Fig. 3b). Then, we examined the $\mathrm{O}_{2}$-absorbing capacity of TPZ/PFA@UiO-66@ PDA NPs in vitro. 143B cells were cultured with different treatments for $3 \mathrm{~h}$. A hypoxia probe was used to detect enhanced cellular hypoxia. Flow cytometry and quantitative analysis showed significantly more pimonidazole hydrochloride-positive cells in the TPZ/PFA@UiO-66@ PDA and TPZ/PFA@UiO-66@PDA+ laser groups, confirming that TPZ/PFA@UiO-66@PDA NPs induced cellular hypoxia (Fig. 3c and e). To explore whether the TPZ/PFA@UiO-66@PDA NPs could improve the efficacy of tumor treatment, Annexin V/propidium iodide (PI) apoptosis detection and live/dead assays were performed to measure the apoptosis-inducing capability in vitro. Flow cytometry analysis showed that more apoptotic cells were found in the TPZ/PFA@UiO-66@PDA and TPZ/PFA@UiO-66@PDA+ laser groups (Fig. 3d, g). Cells treated with TPZ/PFA@UiO-66@PDA and TPZ/ PFA@UiO-66@PDA + laser displayed strong red fluorescence, whereas the remaining groups showed relatively weak red fluorescence (Fig. 3f). Additional file 7: Fig. S6 also demonstrated tumor cell growth was inhibited in the TPZ/PFA@UiO-66@PDA and TPZ/PFA@UiO-66@ PDA+laser groups using Cell Counting Kit-8 method.

\section{TPZ/PFA@UiO-66@PDA upregulated} the oxygen-dependent HIF-1 a pathway and decreased $\mathrm{O}_{2}$ in tumor tissues

To explore the treatment mechanism, 143B cells were grouped as follows: Control, TPZ, TPZ@UiO-66, and TPZ/PFA@UiO-66@PDA. After 3 h of incubation, RNA was extracted for RT-PCR analysis. Compared to the control group, HIF- $1 \alpha$, HIF- $1 \beta$, p300, and CBP in the oxygen-dependent HIF- $1 \alpha$ pathway were upregulated in the TPZ/PFA@UiO-66@PDA group, as were proapoptotic protein genes in the BCL-2 family (BAX, BAK, BID) and Caspase-3 (Fig. 4a). Western blotting showed that HIF-1 $\alpha$ expression in the TPZ/PFA@UiO-66@PDAtreated group was higher than that in the control group (Fig. 4b). Immunohistochemistry of the hypoxyprobe revealed more positive cells in the TPZ/PFA@UiO-66@ PDA-treated group, which also confirmed the enhanced cellular hypoxia (Fig. 4c). Next, we performed HIF-1 $\alpha$ and Caspase- 3 staining after $3 \mathrm{~h}$ of incubation. HIF- $1 \alpha$ and Caspase- 3 fluorescence was significantly stronger in the TPZ/PFA@UiO-66@PDA-treated group than in the other groups (Fig. 4d). Additional file 8: Fig. S7 showed 143B cells treated with $808 \mathrm{~nm}$ laser demonstrated no upregulation of the oxygen-dependent HIF- $1 \alpha$ pathway (HIF- $1 \alpha$, HIF-1 $\beta$, p300, CBP) at the RNA level, and no upregulation of HIF- $1 \alpha$ at the protein level. To study the in vivo efficacy of TPZ/PFA@UiO-66@PDA-induced hypoxia enhancement, $100 \mu \mathrm{L}$ of TPZ/PFA@UiO-66@ PDA NPs was intratumorally injected into 143B tumorbearing mice. Photoacoustic images (Fig. 4e) showed that the average $\mathrm{O}_{2}$ content of tumor tissues continued to decrease after treatment with TPZ/PFA@UiO-66@PDA NPs for $0.5,1,2$, and $3 \mathrm{~h}$. In vivo photoacoustic imaging demonstrated that no decrease of $\mathrm{O}_{2}$ level was observed in the $808 \mathrm{~nm}$ laser treated subcutaneous tumor mice models (Additional file 8: Fig. S7). Twenty-four hours after injection, the mice were sacrificed, and the tumors were sectioned for HIF- $1 \alpha$ staining. HIF- $1 \alpha$ fluorescence was significantly stronger in the TPZ/PFA@UiO-66@ PDA-injected group than in the saline group (Fig. 4f).

\section{TPZ/PFA@UiO-66@PDA can suppress tumor growth effectively in vivo}

To evaluate the targeting ability and biodistribution of TPZ/PFA@UiO-66@PDA NPs after intravenous injection, 6-week-old male BALB/c 143B tumor-bearing mice were administered treatment via tail vein injection. After intravenous injection of IR-780-doped NPs into 143B tumor-bearing mice, accumulated fluorescence was observed, and major organs, including the tumor, heart, lung, liver, spleen, and kidney, were removed and

\section{(See figure on next page.)}

Fig. 4 a The relative HIF-1a, HIF-1 $\beta$, p300, CBP, BAX, BAK, BID and Caspase-3 gene expression levels in 143B cells subjected to different treatments for $3 \mathrm{~h}$. b The HIF-1 a protein expression level in 143B cells subjected to different treatments for $3 \mathrm{~h}$. c Immunohistochemistry to detect hypoxia in 143B cells using the hypoxyprobe. $\mathbf{d}$ The expression of HIF-1 a and Caspase- 3 in $143 \mathrm{~B}$ cells subjected to different treatments for $3 \mathrm{~h}$ analyzed by the double immunofluorescence labeling method. e In vivo photoacoustic images and the average oxygen content of tumor tissues at different time points after intratumoral administration of PFA/TPZ@UiO-66@PDA NPs or saline and the corresponding quantitative analysis. f Immunohistochemistry to detect HIF-1 a in tumor sections at $24 \mathrm{~h}$ after intratumoral administration of PFA/TPZ@UiO-66@PDA NPs or saline (scale bar, $50 \mu \mathrm{m})$. Asterisks indicate significant differences $\left({ }^{*} \mathrm{P}<0.05\right.$, ${ }^{* *} \mathrm{P}<0.01,{ }^{* * *} \mathrm{P}<0.001$, $\left.{ }^{* * * *} \mathrm{P}<0.0001\right)$ 
a
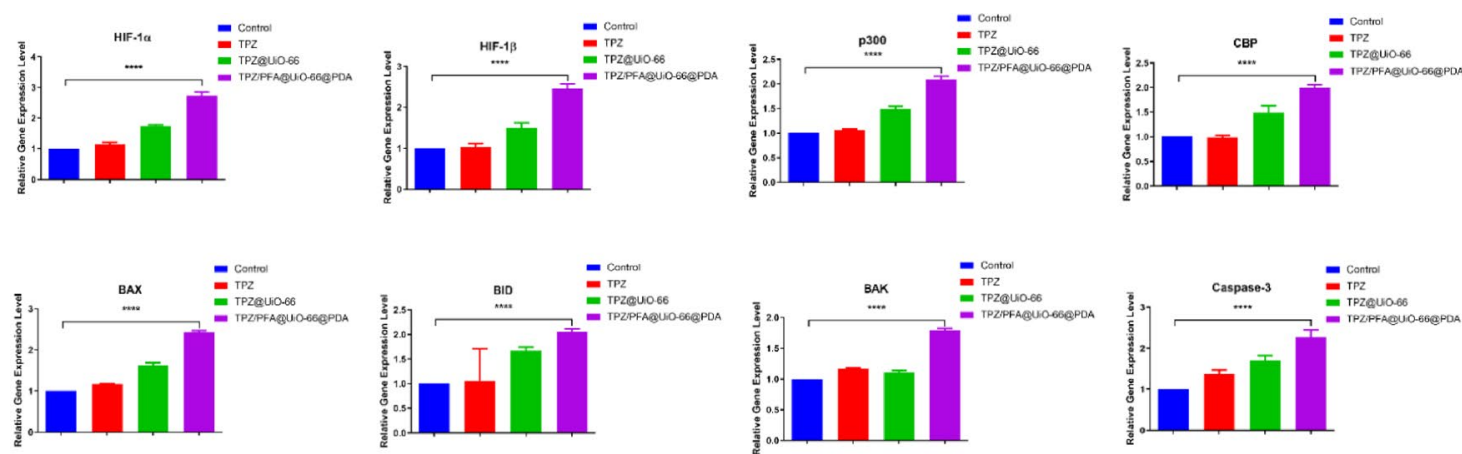

b

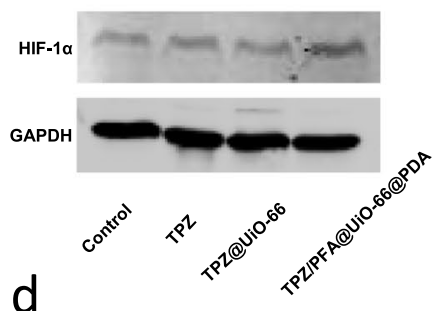

Caspase-3

HIF-1 $\alpha$
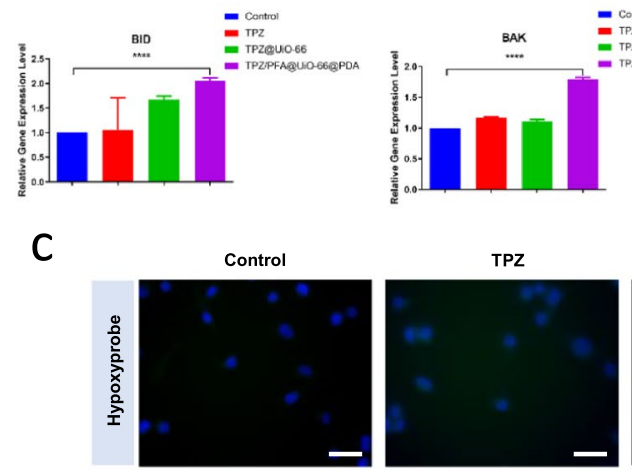

C

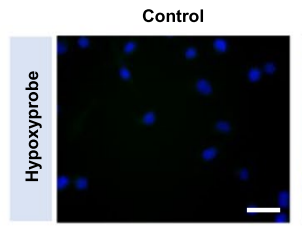

e
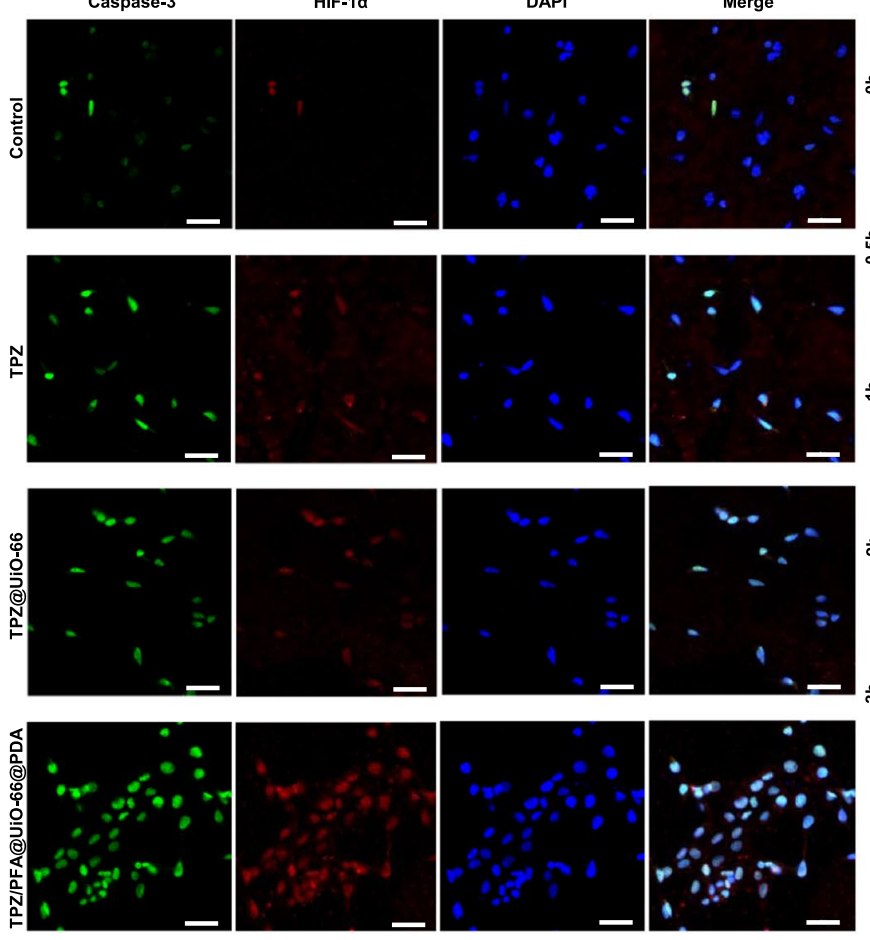

f
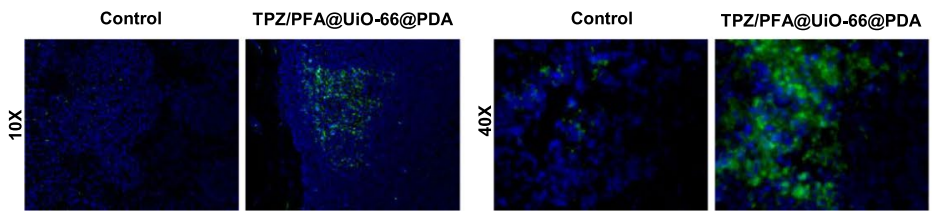

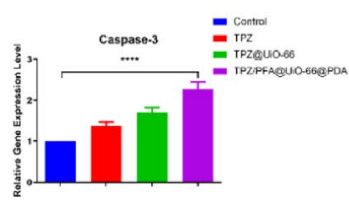

TPZ@UiO-66
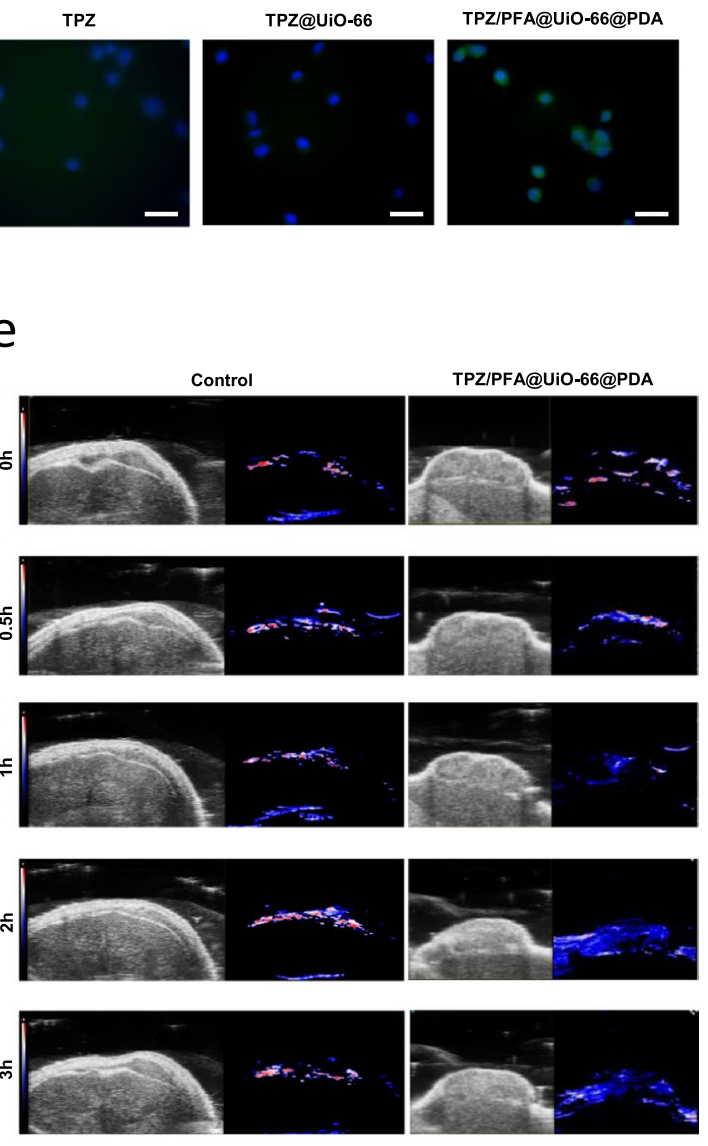

๑-TPZ/PFA@UiO-66@PDA

^ Control

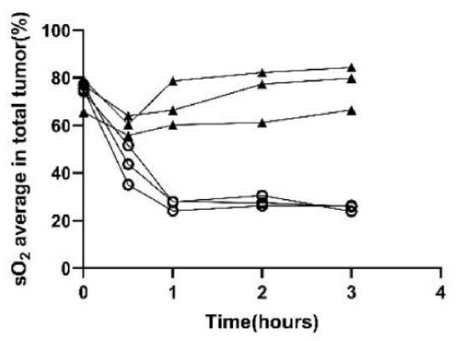

Fig. 4 (See legend on previous page.) 
observed at 1 h, 3 h, 6 h, 12 and 24 h. As shown in Fig. 5a, NPs accumulated at the tumor site $24 \mathrm{~h}$ after injection due to the EPR effect, and the fluorescence intensity in the tumors increased. Next, we studied the photothermal effect of TPZ/PFA@UIO-66@PDA NPs both in vitro and in vivo. The temperature of $500 \mu \mathrm{L}$ of NPs $(0.1 \mathrm{~g} / \mathrm{mL})$ increased to $60.27 \pm 3.02{ }^{\circ} \mathrm{C}$ after 5 min under $808 \mathrm{~nm}$ laser irradiation. After $5 \mathrm{~min}$ under $808 \mathrm{~nm}$ laser irradiation, the temperature of the tumors in mice receiving a tail vein injection of $100 \mu \mathrm{L}$ of NPs reached $47.67 \pm 0.76{ }^{\circ} \mathrm{C}$, which was higher than that in the control group receiving a tail vein injection of $100 \mu \mathrm{L}$ of saline $\left(38.67 \pm 1.33{ }^{\circ} \mathrm{C}\right)$ (Fig. $5 \mathrm{~b}$ and e). Figure $5 \mathrm{c}$ showed that the luminescence intensity in the subcutaneous tumors in the TPZ/PFA@UiO-66@PDA- and TPZ/PFA@UiO$66 @ P D A+$ laser-treated groups on day 8 and day 16 decreased compared with other groups. Figure $5 \mathrm{f}$ showed that the body weights of the mice in all groups remained within the normal range. The dissection of representative tumors was depicted in Fig. 5d. Smaller tumors were obtained from the TPZ/PFA@UiO-66@PDA and TPZ/PFA@UiO-66@PDA + laser groups, confirming the excellent antitumor ability of the TPZ/PFA@UiO-66@ PDA NPs. The tumor weights in the TPZ/PFA@UiO66@PDA- and TPZ/PFA@UiO-66@PDA+ laser-treated groups were significantly smaller than those in the other groups (Fig. 5 g).

After treatment for 16 days, all mice were sacrificed, and the tumors were harvested and sectioned for $H \& E$ staining and immunohistochemistry of PCNA, CD31, HIF- $1 \alpha$ and pimonidazole. Figure 6 demonstrated that PCNA fluorescence was significantly lower in the tumors treated with TPZ/PFA@UiO-66@PDA and TPZ/PFA@ $\mathrm{UiO}-66 @ \mathrm{PDA}+$ laser, whereas the fluorescence intensity of CD31 showed no difference among the groups. HIF- $1 \alpha$ and pimonidazole fluorescence was significantly stronger in the TPZ/PFA@UiO-66@PDA- and TPZ/PFA@UiO66@PDA + laser-treated groups, which indicated that TPZ/PFA@UiO-66@PDA NPs enhanced the hypoxic tumor environment but did not promote angiogenesis (Fig. 7). Moreover, these NPs showed excellent biocompatibility, since no obvious physiological morphological changes were observed in the major organs (liver, heart, lung, spleen and kidney) in any of the groups (Fig. 8), and various blood tests were within normal ranges (Fig. 9).

\section{Discussion}

Hypoxia is a common feature in fast-growing solid tumors that hinders the effect of oxygen-dependent therapy, such as photodynamic therapy (PDT) and sonodynamic therapy (SDT), because PDT/SDT is highly dependent on the oxygen level and limited reactive species can be generated under hypoxia. Two major strategies have been developed to alleviate tumor hypoxia: increasing the $\mathrm{O}_{2}$ supply or reducing $\mathrm{O}_{2}$ consumption. However, most of these solutions have unsatisfactory therapeutic effects due to low intratumoral drug penetration. As the PA imaging in this study indicates, the rich blood supply around the tumor and insufficient vascularization inside the tumor tissue restrict these drugs to the normoxic tumor periphery rather than the hypoxic core.

Cancer starvation therapy is the concept of depriving tumors of nutrient supply to suppress tumor growth. In parallel with conventional vascular occlusion methods, intratumoral glucose/oxygen depletion nanotechnology has emerged and developed rapidly. For example, magnesium silicide NPs can remove intratumoral oxygen efficiently to starve cancer cells [35]. Shan et al. [36] constructed an antioxidant nanocarrier coencapsulating glucose oxidase (GO) and TPZ, which can create tumor hypoxia to convert TPZ into toxic radicals via rapid oxygen depletion while protecting normal tissues by scavenging GOx-catalyzed aerobic glycometabolism byproducts. Perfluorocarbon (PFC) is a promising artificial blood substitute. PFC is also a tumor-specific oxygen carrier due to its excellent oxygen solubility and has been used to relieve tumor hypoxia and enhance the therapeutic effect of PDT/SDT [37]. Liang et al. [38] demonstrated that PFC@porphyrin NPs could serve as an important oxygen reservoir and relieve tumor hypoxia to enhance PDT. Sun et al. [39] developed a mesenchymal stem cell membrane-functionalized liposomal formulation of the sonosensitizer verteporfin and oxygen-loaded PFC, resulting in enhanced SDT therapeutic effects. Although PFC has a high affinity for oxygen, its oxygen release efficacy is quite low. In this study, PFC was used as an intratumoral oxygen-consuming material rather than

\footnotetext{
(See figure on next page.)

Fig. 5 a In vivo fluorescence images of tumor-bearing mice and ex vivo tissue imaging after intravenous injection of IR780-doped PFA/TPZ@ UiO-66@PDA NPs at different time points. He: heart, Li: liver, Sp: spleen, Lu: lung, Ki: kidney and Tu: tumor. b Temperature change and e the quantitative results at different time points in PFA/TPZ@UiO-66@PDA NPs and 143B tumor-bearing mice at $24 \mathrm{~h}$ after intravenous injection of saline or under laser irradiation ( $\left.5 \mathrm{~min}, 808 \mathrm{~nm}, 1.5 \mathrm{~W} \mathrm{~cm}^{-2}\right)(\mathrm{n}=3)$. A, B, and C represent PFA/TPZ@UiO-66@PDA NPs, 143B tumor-bearing mice at 24 h after intravenous injection of PFA/TPZ@UiO-66@PDA NPs and 143B tumor-bearing mice at 24 h after intravenous injection of saline, respectively. c In vivo images of the subcutaneous tumor luciferase signal on day 0, day 8 and day 16. d Photographs of 143B tumor tissues in different groups after 16 days of treatment. $\mathbf{f}$ Body weight and $\mathbf{g}$ tumor weight of mice in different groups. Asterisks indicate significant differences ( ${ }^{*} \mathrm{P}<0.05$, ${ }^{* *} \mathrm{P}<0.01$, $\left.{ }^{* *} \mathrm{P}<0.001,{ }^{* * * *} \mathrm{P}<0.0001\right)$
} 

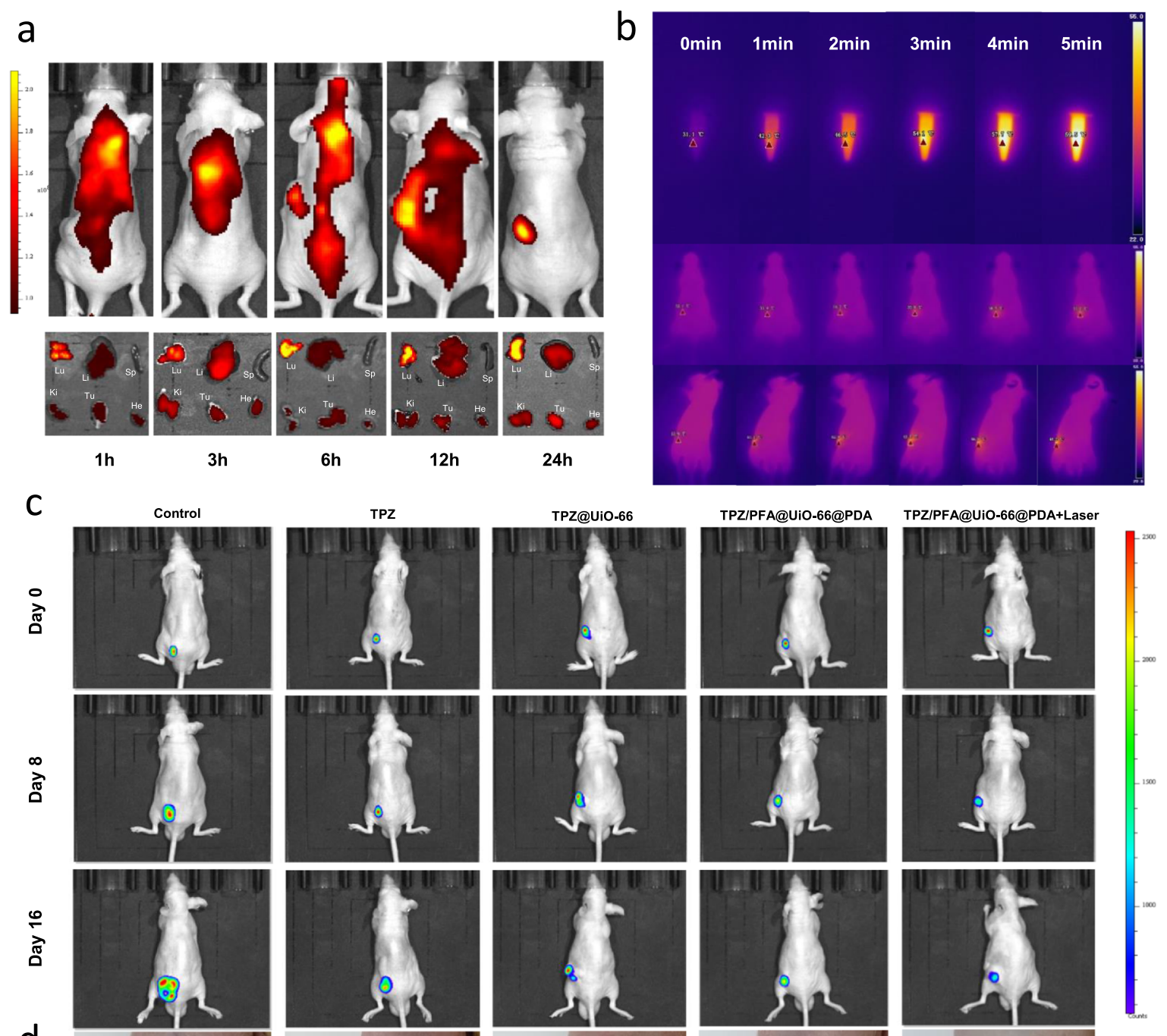

d
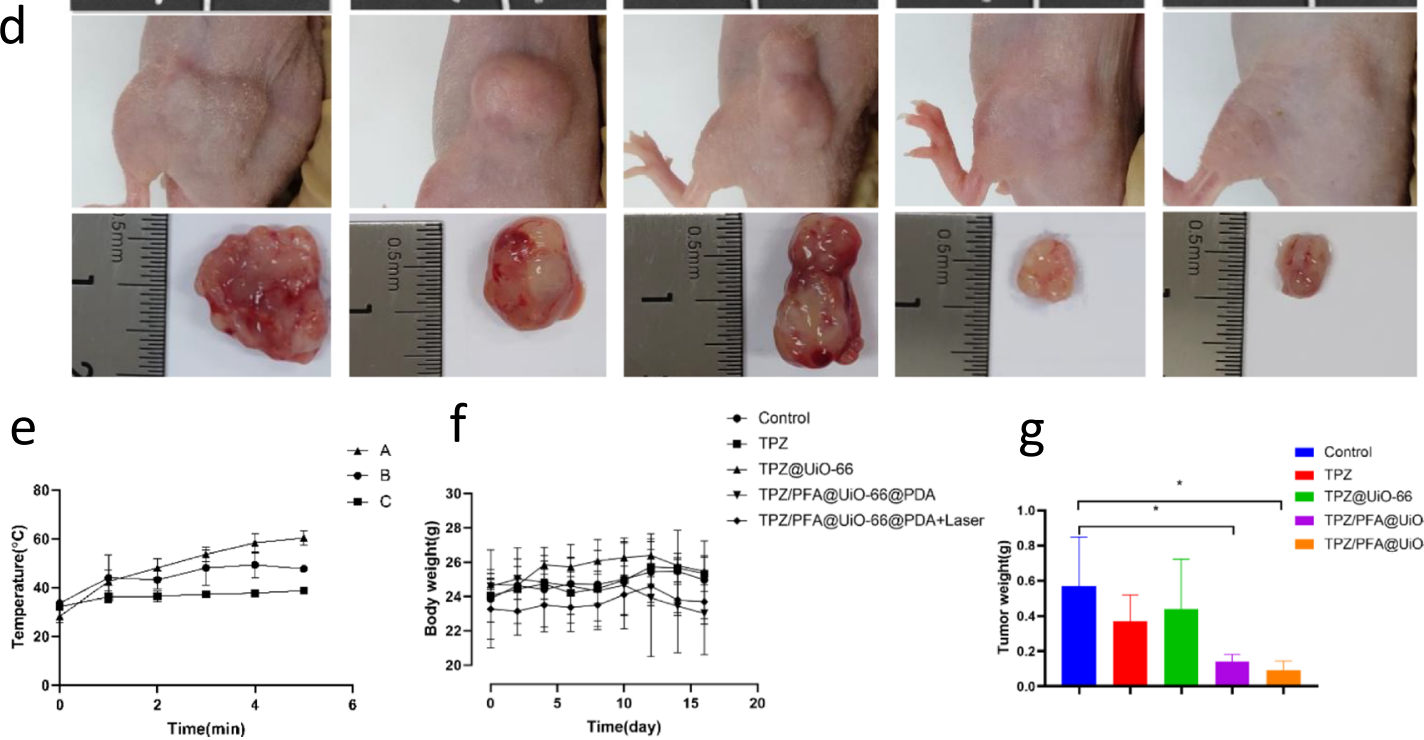

- Control

- TPZ@UiO-66

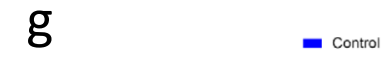

TPZIPFA@UiO-66@PDA

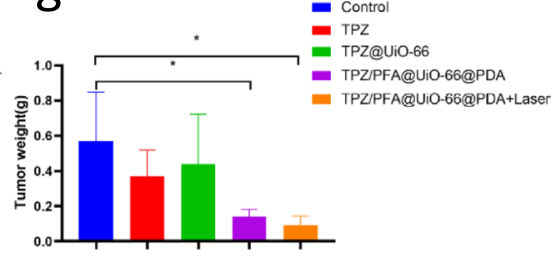

Fig. 5 (See legend on previous page.) 


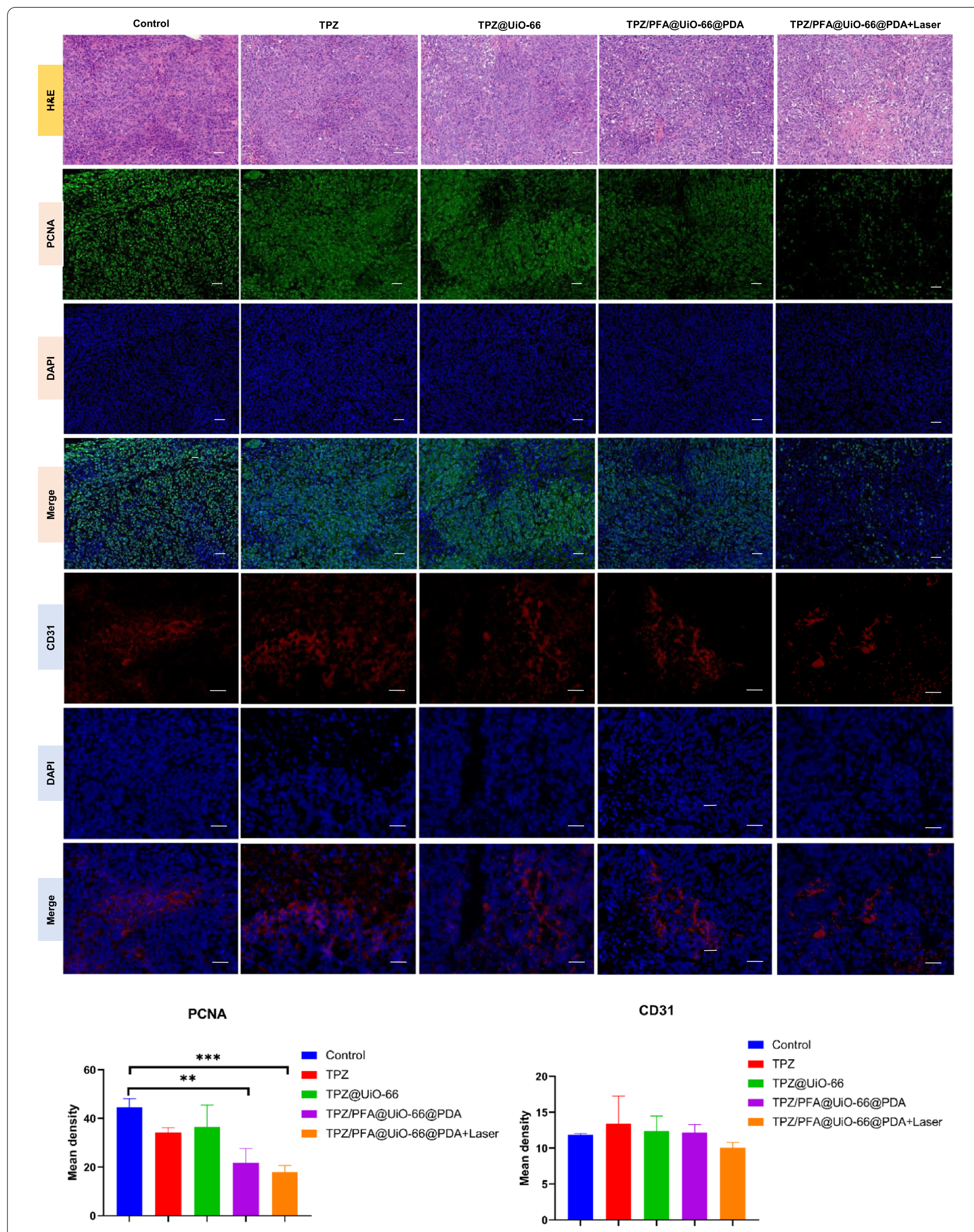

Fig. 6 H\&E staining of subcutaneous xenograft tumors and the expression of PCNA and CD31 in nude mouse tumor specimens in different groups analyzed by immunofluorescence (scale bar, $100 \mu \mathrm{m}$ ). Asterisks indicate significant differences ${ }^{*} P<0.05,{ }^{* *} P<0.01,{ }^{* * *} P<0.001,{ }^{* * * *} P<0.0001$ ) 


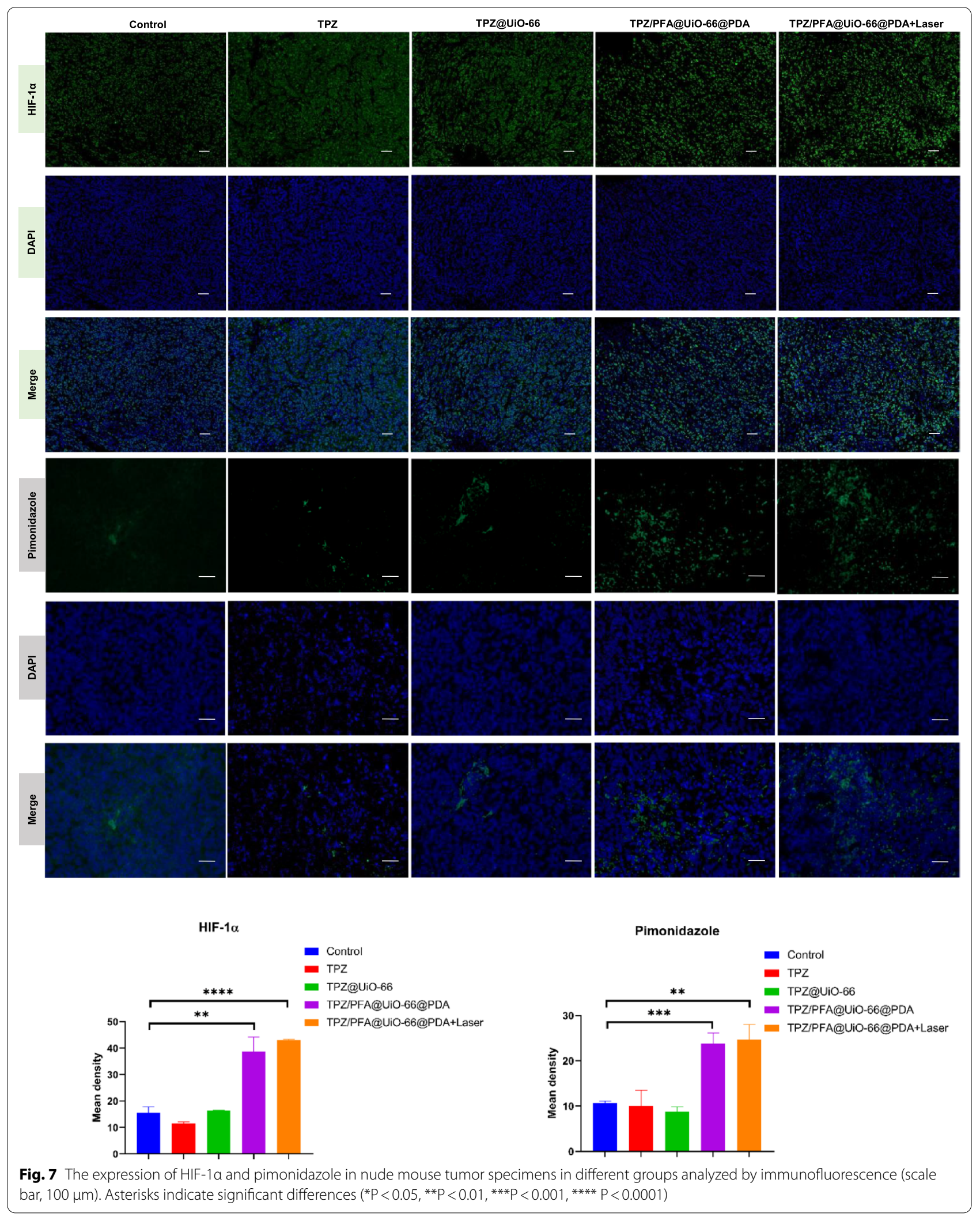




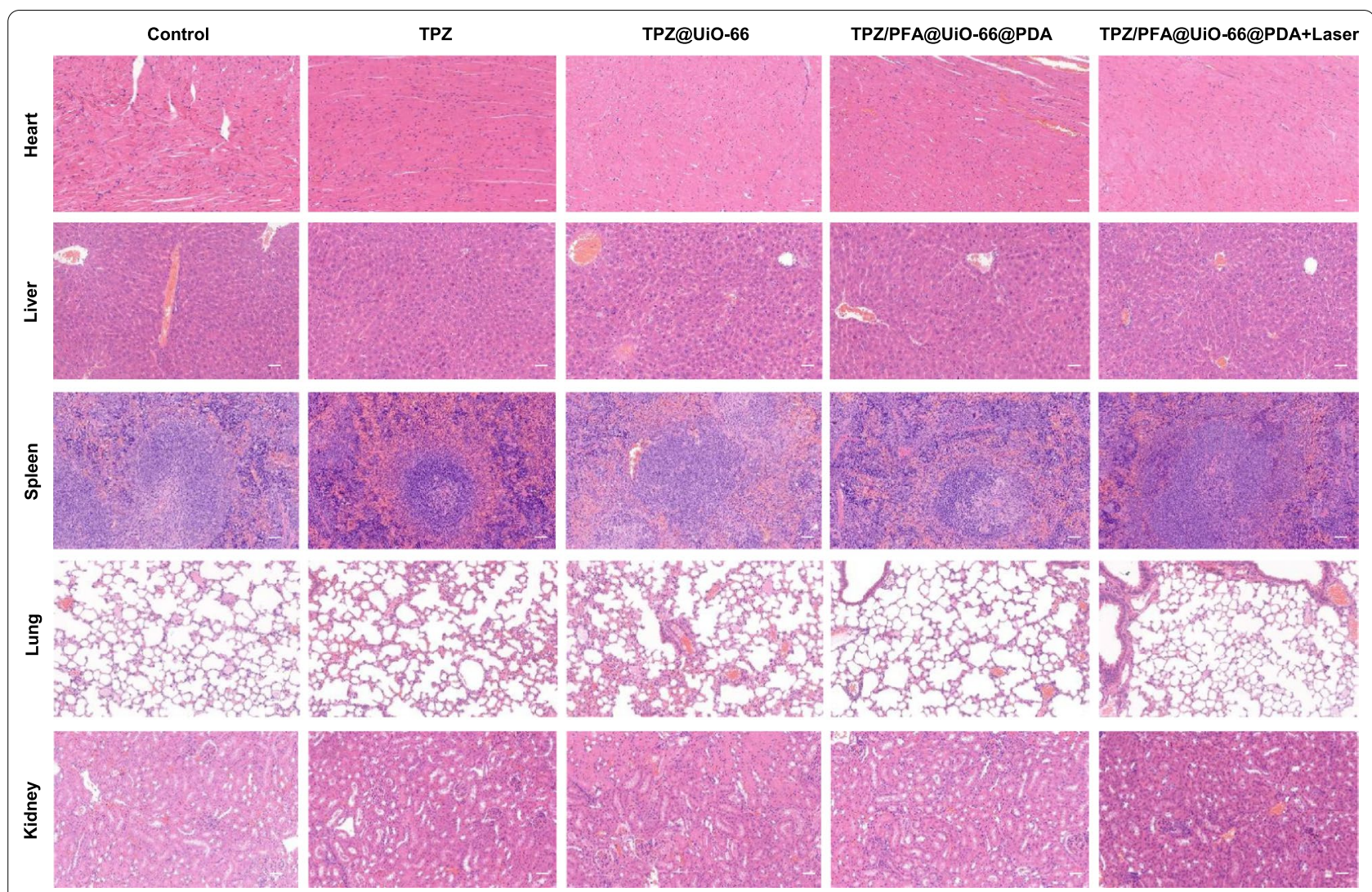

Fig. $8 \mathrm{H} \&$ E staining of the major organs acquired from 143B tumor-bearing mice on day 16 with different treatments (scale bar, $100 \mu \mathrm{m}$ )

an oxygen delivery material. In addition, Wang et al. [16] reported that PFC NPs can be used to create a long-lasting, hypoxic intratumoral environment to enhance the effect of hypoxia-based prodrugs. MOF NPs are characterized by their high porosity, large surface area and high thermal stability. UiO-66 is a porous zirconium-based MOF with open octahedral and tetrahedral cavities [40], and it has been developed for many applications, such as drug delivery systems [41-43], biosensors [44, 45], gas/heavy metal absorbents [33, 46], and biomedicine. In this study, we used UiO-66 as a drug-loading platform by coencapsulating PFA and TPZ, and a PDA coating was added to prevent premature drug leakage.

Notably, it is widely accepted that tumor hypoxia can promote tumor neovascularization and metastasis. In this study, CD31 was not upregulated in groups 4 and 5 . However, whether aggravated tumor hypoxia promotes angiogenesis in the long term remains unknown. Moreover, $143 \mathrm{~B}$ is not a highly metastatic cell line, and it is necessary to repeat this study with a highly metastatic osteosarcoma cell line to confirm that a tumor deoxygenation treatment strategy would not accelerate malignant tumor metastasis.
Second, large numbers of NPs face immune clearance when injected intravenously. Various surface functionalization methods have been explored to enhance the utility of NPs. For example, Zhang et al. [27] reported an erythrocyte membrane-cloaked MOF-based nanomaterial to reduce NP elimination by the immune system. Sun et al. [39] constructed a mesenchymal stem cell membranefunctionalized biomimetic sonosensitizer, which increased circulation and targeting efficacy. Further studies should focus on enhancing the permeability and retention effect of nanomaterials in the tumor while reducing immune clearance. Another challenge is the limited penetration of 808 $\mathrm{nm}$ laser light, as osteosarcoma is a nonsuperficial disease. Some progress has been made toward solving this problem, including strategies such as converting near-infrared rays into photodynamic light in situ [47-50] and taking advantage of aggregation-induced emission photosensitizers to achieve strong radiotherapy and radiodynamic therapy effects under X-ray irradiation [51]. In addition, strong fluorescence intensity was observed in the lungs $24 \mathrm{~h}$ after tail vein injection, which could be a concern in this study. 

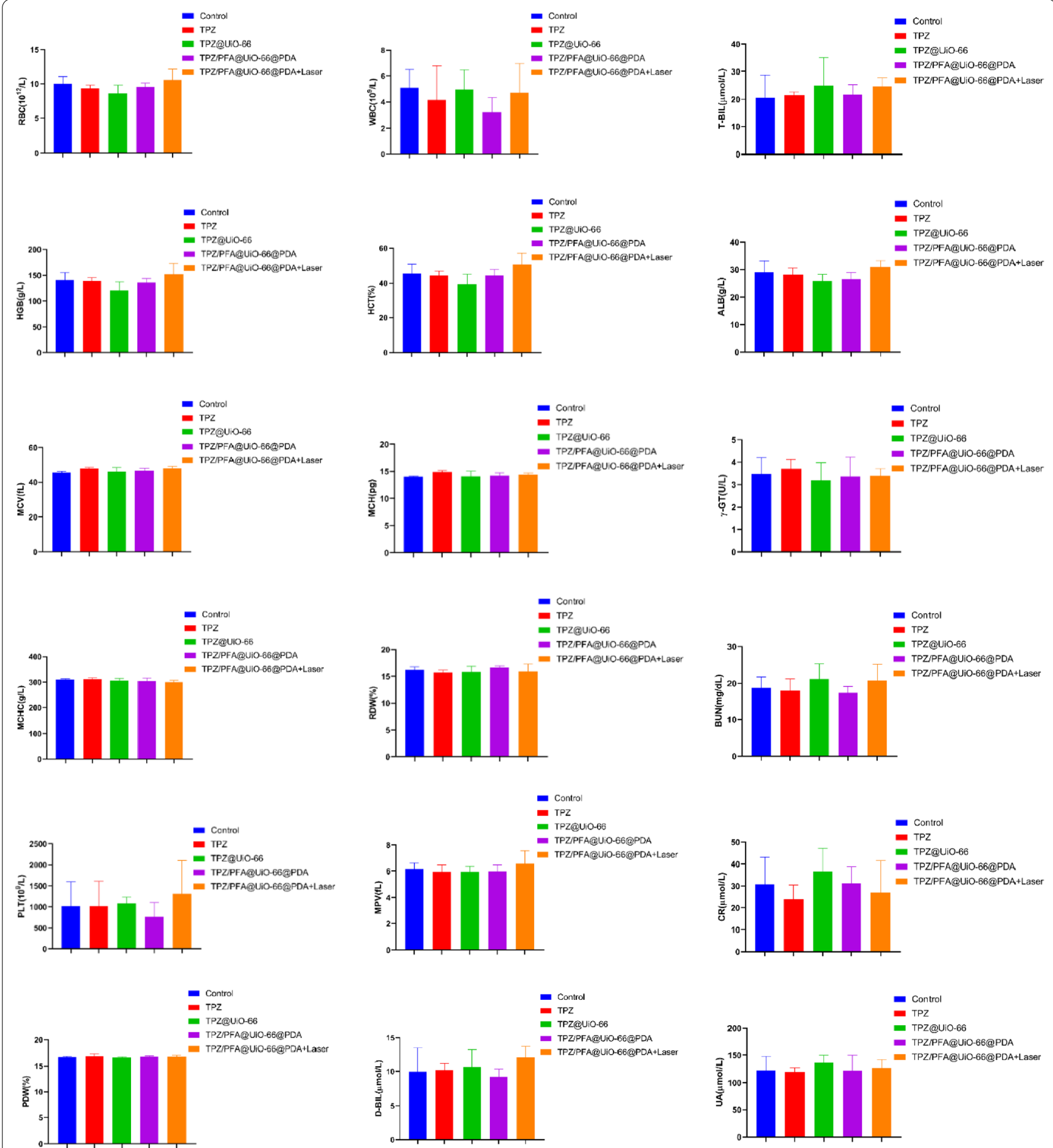

Fig. 9 Blood biochemical levels and hematological parameters in 143B tumor-bearing mice on day 16 after different treatments

\section{Conclusions}

In summary, we successfully constructed biocompatible TPZ/PFA@UiO-66@PDA NPs to suppress tumor growth by enhancing the hypoxic tumor environment. Once injected into the tail vein, TPZ/PFA@UiO-66@PDA NPs accumulated in the tumor region via the EPR effect. After uptake by tumor cells, TPZ/PFA@UiO-66@PDA NPs were degraded to release PFA, TPZ and PDA. Then, PFA upregulated the oxygen-dependent HIF-1 $\alpha$ pathway and induced apoptosis by enhancing the hypoxic tumor environment. TPZ transformed to toxic BTZ and was activated by enhanced tumor hypoxia. PTT was utilized to further 
enhance drug-resistant tumor cell killing. Consequently, tumor growth was significantly suppressed (Additional files $1,2,3,4,5,6,7,8)$.

\begin{abstract}
Abbreviations
PFA: Perfluorotributylamine; TPZ: Tirapazamine; PDA: Polydopamine; MOF: Metal organic framework; PTT: Photothermal therapy; BTZ: Benzotriazinyl; XRD: X-ray diffraction; SEM: Scanning electron microscopy; TEM: Transmission electron microscopy; TGA: Thermogravimetric analysis; DMEM: Dulbecco's minimum essential medium; GAPDH: Glyceraldehyde 3-phosphate dehydrogenase 1; PA: Photoacoustic; H\&E: Hematoxylin-eosin; SD: Standard deviation; ANOVA: Oneway analysis of variance; PDT: Photodynamic therapy; SDT: Sonodynamic therapy; GO: Glucose oxidase; PFC: Perfluorocarbon; EPR effect: Enhanced permeability and retention effect.
\end{abstract}

\section{Supplementary Information}

The online version contains supplementary material available at https://doi.org/ 10.1186/s12951-021-01013-0.

\section{Additional file 1. Supplementary Methods. \\ Additional file 2: Fig. S1. PXRD spectra of UiO-66. \\ Additional file 3: Fig. S2. SEM and TEM images ofTPZ/PFA@UiO-66@PDA.}

Additional file 4: Fig. S3. SEM images of TPZ/PFA@UiO-66@PDA dispersed in culture media or PBS for $24 \mathrm{~h}$.

Additional file 5: Fig. S4. The standard calibration curve of TPZ and the in vitro release curve of TPZ from TPZ@UiO-66 and TPZ/PFA@UiO-66@PDA.

Additional file 6: Fig. S5. Cytotoxicity of UiO-66 in 143B and R-BMSC after co-incubation for 3 days.

Additional file 7: Fig. S6. Cell viability of 143B cells after incubation with different treatments for 3 days.

Additional file 8: Fig. S7. Influence of the $808 \mathrm{~nm}$ laser on the oxygendependent HIF-1a pathway in vitro at the RNA and protein level and the average oxygen content of tumor tissues in vivo

\section{Acknowledgements}

Not applicable.

\section{Authors' contributions}

$Z Z$, JW, YM and HT designed the study. HC, YF, KF, YZ, XW, HH, YC, WW and YX performed the laboratory correlative experiments. HC, YF, and KF analyzed data. $\mathrm{HC}$ wrote the manuscript. YF and KF edited the manuscript. All authors read and approved the final manuscript.

\section{Funding}

This work was supported by National Key R\&D Program of China (2018YFB 1105600/2018YFA0703000), National Natural Science Foundation of China (82072412/81772326), Project of Shanghai Science and Technology Commission (19XD1434200/18431903700), Two-hundred Talent Support (20152224), National infrastructures for translational medicine (Shanghai) open grant (Grant No:TMSK-2020-120)

\section{Availability of data and materials}

All data generated or analyzed during this study are included in this manuscript.

\section{Declarations}

\section{Ethics approval and consent to participate}

All animal experiments were conformed to the guidelines and standards of the Experimental Animal Center of Shanghai Ninth People's Hospital, Shanghai Jiao Tong University School of Medicine.
Consent for publication

All authors agree to publish this manuscript.

\section{Competing interests}

The authors declare no competing interests.

\section{Author details}

${ }^{1}$ Shanghai Key Laboratory of Orthopaedic Implants, Department of Orthopaedic Surgery, Shanghai Ninth People's Hospital, Shanghai Jiao Tong University School of Medicine, Shanghai 200011, China. ${ }^{2}$ Department of Oral \& Maxillofacial - Head \& Neck Oncology, Shanghai Ninth People's Hospital, Shanghai Jiao Tong University School of Medicine; College of Stomatology, Shanghai Jiao Tong University; National Center for Stomatology; National Clinical Research Center for Oral Diseases; Shanghai Key Laboratory of Stomatology, Shanghai, China. ${ }^{3}$ Institute of Microsurgery on Extremities, Department of Orthopedic Surgery, Shanghai Jiaotong University Affiliated Sixth People's Hospital, Shanghai, China. ${ }^{4}$ College of Medicine, Southwest Jiaotong University, Chengdu, China. ${ }^{5}$ School of Biomedical Engineering, Shanghai Jiao Tong University, Shanghai, China.

Received: 21 June 2021 Accepted: 25 August 2021

Published online: 30 September 2021

\section{References}

1. Zhang L, Wang S, Wang Y, Zhao W, Zhang Y, Zhang N, Xu H. Effects of hypoxia in intestinal tumors on immune cell behavior in the tumor microenvironment. Front Immunol. 2021;12:645320.

2. Lu J, Tan M, Cai Q. The Warburg effect in tumor progression: mitochondrial oxidative metabolism as an anti-metastasis mechanism. Cancer Lett. 2015;356(2 Pt A):156-64.

3. Meng L, Xing Z, Guo Z, Qiu Y, Liu Z. Hypoxia-induced microRNA-155 overexpression in extracellular vesicles promotes renal cell carcinoma progression by targeting FOXO3. Aging. 2021;13(7):9613-26.

4. Song JS, Chang CC, Wu CH, Dinh TK, Jan JJ, Huang KW, Chou MC, Shiue TY, Yeh KC, Ke YY, Yeh TK, Ta YN, Lee CJ, Huang JK, Sung YC, Shia KS, Chen Y. A highly selective and potent CXCR4 antagonist for hepatocellular carcinoma treatment. Proc Natl Acad Sci USA. 2021;118(13):e2015433118. doi:https:// doi.org/10.1073/pnas.2015433118.

5. Su H, Yang F, Fu R, Li X, French R, Mose E, Pu X, Trinh B, Kumar A, Liu J, et al. Cancer cells escape autophagy inhibition via NRF2-induced macropinocytosis. Cancer Cell. 2021;39(5):678-93.e11. doi:https://doi.org/10.1016/j.ccell. 2021.02.016.

6. Zheng D, Li B, Xu L, Zhang QL, Fan JX, Li CX, Zhang XZ. Normalizing tumor microenvironment based on photosynthetic abiotic/biotic nanoparticles. ACS Nano. 2018;12(6):6218-27.

7. Xu S, Zhu X, Zhang C, Huang W, Zhou Y, Yan D. Oxygen and Pt(II) self-generating conjugate for synergistic photo-chemo therapy of hypoxic tumor. Nat Commun. 2018;9(1):2053.

8. Zhao LP, Zheng RR, Chen HQ, Liu LS, Zhao XY, Liu HH, Qiu XZ, Yu XY, Cheng H, Li SY. Self-delivery nanomedicine for O2-economized photodynamic tumor therapy. Nano Lett. 2020;20(3):2062-71.

9. Min Q, Liang T, Zhang B, Xing Z, Dong Y, Xu H, Chen X, Jiang L, Zhu JJ. Adapting and remolding: orchestrating tumor microenvironment normalization with photodynamic therapy by size transformable nanoframeworks. Angew Chem Int Ed Engl. 2021;60(20):11464-73.

10. Fang H, Gai Y, Wang S, Liu Q, Zhang X, Ye M, Tan J, Long Y, Wang K, Zhang $Y$, Lan X. Biomimetic oxygen delivery nanoparticles for enhancing photodynamic therapy in triple-negative breast cancer. J Nanobiotechnol. 2021;19(1):81

11. Ge L, Qiao C, Tang Y, Zhang X, Jiang X. Light-activated hypoxia-sensitive covalent organic framework for tandem-responsive drug delivery. Nano Lett. 2021;21(7):3218-24.

12. Li Y, Sutrisno L, Hou Y, Fei Y, Xue C, Hu Y, Li M, Luo Z. A redox-activatable biopolymer-based micelle for sequentially enhanced mitochondriatargeted photodynamic therapy and hypoxia-dependent chemotherapy. Chem Commun (Camb). 2020;56(69):9978-81.

13. Zheng L, Wang B, Sun Y, Dai B. An oxygen-concentration-controllable multiorgan microfluidic platform for studying hypoxia-induced lung cancer-liver metastasis and screening drugs. ACS Sens. 2021;6(3):823-32. 
14. Xin F, Wu M, Cai Z, Zhang X, Wei Z, Liu X. Tumor microenvironment triggered cascade-activation nanoplatform for synergistic and precise treatment of hepatocellular carcinoma. Adv Healthc Mater; 2021;10(10):e2002036. https://doi.org/10.1002/adhm.202002036. Epub 2021 Feb 28.

15. Lee D, Jang SY, Kwon S, Lee Y, Park E, Koo H. Optimized combination of photodynamic therapy and chemotherapy using gelatin nanoparticles containing tirapazamine and pheophorbide a. ACS Appl Mater Interfaces. 2021;13(9):10812-21 .

16. Wang W, Cheng Y, Yu P, Wang H, Zhang $Y, X u H$, Ye Q, Yuan A, Hu Y, Wu J. Perfluorocarbon regulates the intratumoural environment to enhance hypoxia-based agent efficacy. Nat Commun. 2019;10(1):1580.

17. Eddaoudi M, Moler DB, Li H, Chen B, Reineke TM, O'Keeffe M, Yaghi OM. Modular chemistry: secondary building units as a basis for the design of highly porous and robust metal-organic carboxylate frameworks. Acc Chem Res. 2001;34(4):319-30.

18. Zadvarzi SB, Khavarpour M, Vahdat SM, Baghbanian SM, Rad AS. Synthesis of Fe304@chitosan@ZIF-8 towards removal of malachite green from aqueous solution: Theoretical and experimental studies. Int J Biol Macromol. 2021;168:428-41.

19. Férey G. Hybrid porous solids: past, present, future. Chem Soc Rev. 2008;37(1):191-214

20. Li Q, Guo J, Zhu H, Yan F. Space-confined synthesis of ZIF-67 nanoparticles in hollow carbon nanospheres for CO2 adsorption. Small. 2019;15(8):e1804874.

21. Connolly BM, Aragones-Anglada M, Gandara-Loe J, Danaf NA, Lamb DC, Mehta JP, Vulpe D, Wuttke S, Silvestre-Albero J, Moghadam PZ, Wheatley AEH, FairenJimenez D. Tuning porosity in macroscopic monolithic metal-organic frameworks for exceptional natural gas storage. Nat Commun. 2019;10(1):2345.

22. Kreno LE, Leong K, Farha OK, Allendorf M, Van Duyne RP, Hupp JT. Metal-organic framework materials as chemical sensors. Chem Rev. 2012;112(2):1105-25.

23. Liu S, Bai J, Huo Y, Ning B, Peng Y, Li S, Han D, Kang W, Gao Z. A zirconium-porphyrin MOF-based ratiometric fluorescent biosensor for rapid and ultrasensitive detection of chloramphenicol. Biosens Bioelectron. 2020;149:111801.

24. Horcajada P, Chalati T, Serre C, Gillet B, Sebrie C, Baati T, Eubank JF, Heurtaux D, Clayette P, Kreuz C, Chang JS, Hwang YK, Marsaud V, Bories PN, Cynober L, Gil S, Férey G, Couvreur P, Gref R. Porous metal-organic-framework nanoscale carriers as a potential platform for drug delivery and imaging. Nat Mater. 2010;9(2):172-8.

25. Della Rocca J, Liu D, Lin W. Nanoscale metal-organic frameworks for biomedical imaging and drug delivery. Acc Chem Res. 2011;44(10):957-68.

26. Cao F, Ju E, Liu C, LiW, Zhang Y, Dong K, Liu Z, Ren J. Encapsulation of aggregated gold nanoclusters in a metal-organic framework for real-time monitoring of drug release. Nanoscale. 2017;9(12):4128-34.

27. Zhang L, Wang Z, Zhang Y, Cao F, Dong K, Ren J. Erythrocyte membrane cloaked metal-organic framework nanoparticle as biomimetic nanoreactor for starvationactivated colon cancer therapy. ACS Nano. 2018;12(10):10201-11.

28. Chen TH, Popov I, Kaveevivitchai W, Chuang YC, Chen YS, Jacobson AJ, Miljanić O. Mesoporous fluorinated metal-organic frameworks with exceptional adsorption of fluorocarbons and CFCS. Angew Chem Int Ed Engl. 2015;54(47):13902-6.

29. Shen J, He X. Simultaneous interlayer and intralayer space control in twodimensional metal-organic frameworks for acetylene/ethylene separation. Nat Commun. 2020;11(1):6259.

30. Zhao Y, Zhao T, Cao Y, Sun J, Zhou Q, Chen H. Temperature-sensitive lipid-coated carbon nanotubes for synergistic photothermal therapy and gene therapy. ACS Nano. 2021;15(4):6517-29.

31. Prabhu SM, Chuaicham C, Park CM, Jeon BH, Sasaki K. Synthesis and characterization of defective UiO-66 for efficient co-immobilization of arsenate and fluoride from single/binary solutions. Environ Pollut. 2021;278:116841.

32. Shen $L$, Wu W, Liang $R$, Lin $R$, Wu L. Highly dispersed palladium nanoparticles anchored on UiO-66(NH2) metal-organic framework as a reusable and dual functional visible-light-driven photocatalyst. Nanoscale. 2013;5(19):9374-82.

33. Noraee Z, Jafari A, Ghaderpoori M, Kamarehie B, Ghaderpoury A. Use of metal-organic framework to remove chromium (VI) from aqueous solutions. J Environ Health Sci Eng. 2019;17(2):701-9.

34. Yao BJ, Jiang WL, Dong Y, Liu ZX, Dong YB. Post-synthetic polymerization of UiO-66-NH2 nanoparticles and polyurethane oligomer toward stand-alone membranes for dye removal and separation. Chemistry. 2016;22(30):10565-71.

35. Zhang C, Ni D, Liu Y, Yao H, Bu W, Shi J. Magnesium silicide nanoparticles as a deoxygenation agent for cancer starvation therapy. Nat Nanotechnol. 2017;12(4):378-86
36. Shan L, Fan W, Wang W, Tang W, Yang Z. Organosilica-based hollow mesoporous bilirubin nanoparticles for antioxidation-activated self-protection and tumor-specific deoxygenation-driven synergistic therapy. ACS Nano. 2019;13(8):8903-16.

37. Gao M, Liang C, Song X, Chen Q, Jin Q, Wang C, Liu Z. Erythrocyte-Membrane-Enveloped Perfluorocarbon as Nanoscale Artificial Red Blood Cells to Relieve Tumor Hypoxia and Enhance Cancer Radiotherapy. Adv Mater; 2017;29(35)

38. Liang X, Chen M, Bhattarai P, Hameed S, Dai Z. Perfluorocarbon@Porphyrin nanoparticles for tumor hypoxia relief to enhance photodynamic therapy against liver metastasis of colon cancer. ACS Nano. 2020;14(10):13569-83.

39. Sun L, Xu Y, Zhang X, Gao Y, Chen J, Zhou A, Lu Q, Wang Z, Shao K, Wu H, et al. Mesenchymal stem cells functionalized sonodynamic treatment for improving therapeutic efficacy and compliance of orthotopic oral cancer. Adv Mater. 2020;32(48):e2005295.

40. Farboudi A, Mahboobnia K, Chogan F, Karimi M, Askari A, Banihashem S, Davaran S, Irani M. UiO-66 metal organic framework nanoparticles loaded carboxymethyl chitosan/poly ethylene oxide/polyurethane core-shell nanofibers for controlled release of doxorubicin and folic acid. Int J Biol Macromol. 2020;150:178-88.

41. Gu C, Bai L, Pu L, Gai P, Li F. Highly sensitive and stable self-powered biosensing for exosomes based on dual metal-organic frameworks nanocarriers. Biosens Bioelectron. 2021;176:112907.

42. Rabiee N, Bagherzadeh M. Polymer-coated NH(2)-UiO-66 for the codelivery of DOX/pCRISPR. ACS Appl Mater Interfaces. 2021;13(9):10796-811.

43. Jodłowski PJ, Kurowski G, Kuterasiński Ł, Sitarz M, Jeleń P, Jaśkowska J, Kołodziej A, Pajdak A, Majka Z, Boguszewska-Czubara A. Cracking the chloroquine conundrum: the application of defective $\mathrm{UiO}-66$ metal-organic framework materials to prevent the onset of heart defects-in vivo and in vitro. ACS Appl Mater Interfaces. 2021;13(1):312-23.

44. Cheng Y, Lai OM, Tan CP. Proline-modified UIO-66 as nanocarriers to enhance Candida rugosa lipase catalytic activity and stability for electrochemical detection of nitrogen. ACS Appl Mater Interfaces. 2021;13(3):4146-55.

45. Meng T, Shang N, Nsabimana A, Ye H, Wang H, Wang C, Zhang Y. An enzyme-free electrochemical biosensor based on target-catalytic hairpin assembly and Pd@UiO-66 for the ultrasensitive detection of microRNA-21. Anal Chim Acta. 2020;1138:59-68.

46. Daradmare S, Xia M, Le VN, Kim J, Park BJ. Metal-organic frameworks/alginate composite beads as effective adsorbents for the removal of hexavalent chromium from aqueous solution. Chemosphere. 2021;270:129487.

47. Wang Y, Chen L. Smart fluorescent probe strategy for precision targeting hypoxic tumor. J Med Chem. 2021;64(6):2967-70.

48. Li L, Zhang B, Liu Y, Gao R, Zhou J. A spontaneous membrane-adsorption approach to enhancing second near-infrared deep-imaging-guided intracranial tumor therapy. ACS Nano. 2021;15(3):4518-33.

49. Xu C, Jiang Y, Han Y, Pu K. A polymer multicellular nanoengager for synergistic NIR-II photothermal immunotherapy. Adv Mater. 2021;33(14):e2008061.

50. Zou R, Gao Y, Zhang Y, Jiao J, Wong KL. (68)Ga-labeled magnetic-NIR persistent luminescent hybrid mesoporous nanoparticles for multimodal imaging-guided chemotherapy and photodynamic therapy. ACS Appl Mater Interfaces. 2021;13(8):9667-80.

51. Liu J, Hu F, Wu M, Tian L, Gong F, Zhong X, Chen M, Liu Z, Liu B. Bioorthogonal coordination polymer nanoparticles with aggregation-induced emission for deep tumor-penetrating radio- and radiodynamic therapy. Adv Mater. 2021;33(9):e2007888.

\section{Publisher's Note}

Springer Nature remains neutral with regard to jurisdictional claims in published maps and institutional affiliations. 